\title{
SQUIDs for Standards and Metrology
}

John Gallop and François Piquemal

9.1 Introduction 96

9.2 SQUIDs in Voltage Metrology 97

9.2.1 SQUID Femtovoltmeter: Highly Accurate Voltage Measurement 97

9.2.1.1 Principle 97

9.2.1.2 Femtovoltmeter for Verifying the Universality of Josephson Constant 98

9.2.2 SQUID Ampermeter for linking the Volt to Mechanical SI Units 98

9.2.3 Other Precise SQUID-Based Voltage Measurements 100

9.3 Cryogenic Current Comparator (CCC) 101

9.3.1 Principle of the CCC 101

9.3.2 Limits to Accuracy of CCC Ratio Measurements 104

9.3.2.1 Sensitivity and Current Resolution 104

9.3.2.2 Exactness of the CCC Current Ratio 108

9.3.3 Resistance Ratio Measurement 111

9.3.3.1 Introductory 111

9.3.3.2 Resistance Bridge Based on Type I CCC 113

9.3.3.3 Some Remarks on Construction 114

9.3.4 Measurements of Very Low Currents from SET Sources 115

9.3.4.1 Introduction 115

9.3.4.2 Design and Performance of the CCC as an Ultra-Low-Current Amplifier 118

9.3.4.3 Measurements of SET Current Sources 119

9.3.5 Type II CCC for Resistance Measurements at Very High Currents 121

9.3.6 CCC for Non-Invasive Sensing of Charged Particle Beams 122

9.4 Other Current Metrological Applications of SQUIDs 123

9.4.1 Thermometry Using SQUIDs 123

9.4.1.1 Introduction 123

9.4.1.2 Magnetic Susceptibility Measurement for a Secondary Thermometer 124

9.4.1.3 The SQUID as a Sensor for a Resistance Thermometer 125

9.4.1.4 Noise Measurement for a "Primary" Thermometer 125

9.4.2 Radio-Frequency Attenuation with SQUIDs 127

9.5 Future Trends and Conclusion 129

The SQUID Handbook. Vol. II: Applications of SQUIDs and SQUID Systems.

John Clarke and Alex I. Braginski (Eds.)

Copyright (C) 2006 WILEY-VCH Verlag GmbH \& Co. KGaA, Weinheim

ISBN: 3-527-40408-2 


\section{1}

\section{Introduction}

Metrology is the science of precise measurement. SQUIDs, combining the two properties of extraordinary sensitivity together with a periodic response to an input signal which is based on a quantum standard (the flux quantum $\Phi_{0}=h / 2 e$ ), are particularly well suited for metrology applications, and especially for quantum electrical metrology. As simple as a superconducting ring interrupted by one or two Josephson junctions, the working principle of SQUID is based on double quantum phenomena both involving the same quantum $\Phi_{0}$ : the quantization of magnetic flux across the ring and the oscillation of the supercurrent circulating across the junction. The first metrological use of the SQUID as a voltmeter (a null detector in the sub-femtovolt range) for testing the universality of the currentphase relationship of a Josephson junction hardly comes as a surprise (see Section 8.2). The second important application of the SQUID in metrology is its use as a magnetic flux detector combined with a marvellous tool: the cryogenic current comparator (CCC). This is the instrument used in a resistance bridge which has allowed National Metrology Institutes (NMIs) to establish the high reproducibility of the quantum resistance standard based on the quantum Hall effect (QHE) (see Section 9.3.3). The CCC can also operate as an ultra low current amplifier with a very highly accurate gain. CCCs have been developed for measuring the very small current delivered by single electron tunneling (SET) sources, (for example: a few picoamps for a single-electron pump). The aim is firstly to establish if SET devices could generate highly quantized current and therefore if a quantum standard of current could be available especially for amplitudes less than $1 \mathrm{nA}$ (see Section 9.3.4). Then the important issue will be the closure of the quantum metrological triangle with a very high precision, by directly combining the ac Josephson effect (ac JE), QHE and SET.

There are at least two other applications of CCCs in electrical metrology nowadays. The first involves a second type of CCC particularly designed to measure direct currents with an amplitude as high as $100 \mathrm{~A}$ (see Section 9.3.5). The second, which implies a CCC based on the initial concept of a superconducting tube, is devoted to noninvasive sensing of charged particle beams.

Other current applications of SQUIDs in metrology concern thermometry (Section 9.41) and a former metrological application of SQUIDs in the field of microwave measurement which may come back to prominence in the future (Section 
9.42). The wide domain of X-ray and $\gamma$-ray spectrometry where SQUIDs are used for metrological purposes is dealt with in Chapter 8 .

It has to be pointed out that many of the metrological applications presented are still in the development phase and this chapter represents a snapshot in time. The chapter ends with an assessment of future trends (see Section 9.5), especially those of single-particle detection, quantum measurement and the requirements of metrology at the nanometer scale.

\section{2}

\section{SQUIDs in Voltage Metrology}

\subsection{1}

\section{SQUID Femtovoltmeter: Highly Accurate Voltage Measurement}

\subsubsection{Principle}

A SQUID magnetometer may be converted very simply to a highly sensitive femtovoltmeter [1], as already discussed in Section 8.2. It is only necessary to take a coil of inductance $L_{\mathrm{i}}$ and a low resistance $R_{\mathrm{i}}$, which is magnetically coupled to the SQUID loop via the mutual inductance $M_{\mathrm{i}}$ and to apply the voltage $V_{\mathrm{s}}$ to be measured across it as shown in Figure 9.1. The current $I_{\mathrm{i}}$, which flows in the input coil, will apply flux to the SQUID loop. This flux may be then detected in the usual way using the flux-locked loop feedback circuit. Of course the input coil may be made of a superconductor, having essentially zero resistance. Then the steady state current flowing is determined by the voltage applied and the internal resistance of the voltage source.

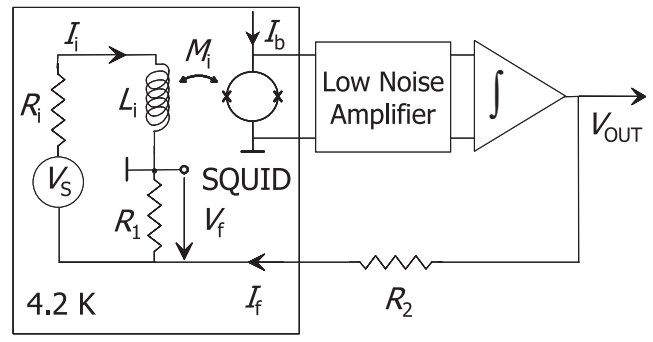

Fig. 9.1 Basic circuit of a picovoltmeter based on a dc SQUID. The feedback current $I_{\mathrm{f}}$ flowing through the resistor $R_{1}$ develops a voltage drop $V_{\mathrm{f}}$ which tends to null $I_{\mathrm{i}}$. Then the signal voltage $V_{\mathrm{S}}$ equals the product $R_{1} I_{\mathrm{f}}$, and the output voltage $V_{\text {OUT }}$ is given by $V_{\text {OUT }}=V_{\mathrm{S}} R_{2} / R_{1}$, typically $R_{2} / R_{1} \gg 1$. $I_{b}$ denotes the SQUID bias current. 


\subsubsection{Femtovoltmeter for Verifying the Universality of Josephson Constant}

In an extreme case, the voltage source may have truly zero resistance. An example would be a Josephson junction irradiated with a spectrally pure microwave source having frequency $f$ and biased on one of the induced Shapiro steps which exhibit quantized voltages at $n \Phi_{0} f$ ( $n$ is an integer), but which have zero differential resistance against small changes in bias current. In this case a steady state current will not be attained: instead an input current increasing linearly in time will be produced at a rate $\mathrm{d} I / \mathrm{d} t=\Delta V_{\mathrm{s}} / L_{\mathrm{i}}$, where $\Delta V_{\mathrm{s}}$ is the constant applied voltage and $L_{\mathrm{i}}$ is the inductance of the input coil [2]. The extreme sensitivity of SQUIDs when used in this way (with vanishingly small input resistance sources) was demonstrated to be at the level of $10^{-22} \mathrm{~V}$ [3]. Tsai et al. used the same principle, where two Josephson junctions made of different materials are biased with the same frequency on the same integer Shapiro step, while they are joined through a superconducting input coil coupled to a SQUID. By attempting to observe the time-dependent signal expected at the SQUID output Tsai et al. were able to put an upper limit on the material dependence of the frequency to voltage ratio [4]. The so defined Josephson constant $K_{J}^{1)}$, is less than 2 parts in $10^{16}$. That strengthens our confidence in the universal aspect of $K_{\mathrm{J}}$ and hence in the equality $K_{\mathrm{J}}=2 e / h$.

Hovewer, even if strong theoretical arguments exist, from a strictly metrological point of view, this relation is not proven. Moreover, this result does not mean an upper deviation of the ratio $K_{\mathrm{J}} /(2 e / h)$ from one is of the order indicated above. The exactness of this relation has been recently tested by the CODATA task group in the framework of the 2002 fundamental constant adjustment [6]. It was shown that there is no significant deviation between $K_{\mathrm{J}}$ and $2 e / h$, but with an uncertainty of 8 parts in $10^{8^{2}}$.

\subsection{2}

\section{SQUID Ampermeter for linking the Volt to Mechanical SI Units}

In the SI system, the electrical quantities are defined so that force and energy generated in electrical systems are measured in the same units as in mechanical systems. For linking the volt and ampere to mechanical SI units, one needs some electromechanical system in which electrical and mechanical energies can be compared directly. Several kinds of experiments contribute to providing values of

1) The determinations of $2 e / h$ in SI units performed in the 1980s, which were found in better agreement than the previous ones, allowed the Comité International des Poids et Mesures (CIPM) to recommend implementing the Josephson effect as a voltage standard, to use the Josephson constant $K_{\mathrm{J}}$ as an estimate of $2 e / h$ and for calibration purposes by assigning to it one single exact value of $K_{\mathrm{I}-90}=483597.9 \mathrm{GHz} / \mathrm{V}$ [5]. Note that the uncertainty on $K_{\mathrm{I}}$ presently given by the CIPM is 4 parts in $10^{7}$ in terms of SI units.
2) This is a fairly large value compared to the agreement of one part in $10^{10}$ routinely found by the NMIs in direct comparisons of $1 \mathrm{~V}$ and $10 \mathrm{~V}$ Josephson voltage standards based on series arrays of large numbers of underdamped junctions (superconductorinsulator-superconductor technology). The arrays contain 2000 to 3000 junctions for $1 \mathrm{~V}$, to more than 10000 junctions for $10 \mathrm{~V}$ (for a review of Josephson standards, see for instance $[7,8])$. 
$K_{\mathrm{J}}$ or $2 e / h$ in SI units (if one assumes the equality $K_{\mathrm{J}}=2 e / h$ to be valid). The moving coil Watt balance is the technique of highest accuracy so far. This method could be the origin of a new SI definition of the kilogram if an uncertainty of one part in $10^{8}$ is reached, the definition possibly based on the Planck constant. A review of Watt balance experiments is given in [9]. Another method, currently investigated by some NMIs with the same objective, involves a SQUID ampermeter [1-13]. This method consists of levitating a superconducting body successively at different equilibrium positions in a non-uniform magnetic field ${ }^{3}$.

Figure 9.2 (a) shows a schematic of the experimental setup currently developed at $\mathrm{NMIJ}^{4)}[10,11]$. A body of small mass $m=25 \mathrm{~g}$, a phosphor-bronze thin wall cone covered with lead, reaches stable levitation positions in the magnetic field induced by the conical superconducting coil. The levitation occurs when the magnetic flux is large enough for the force due to the Meissner effect to balance the weight of the body. The current source is controlled by a SQUID ampermeter to keep always at zero the current $I_{\mathrm{S}}$ in such a way that the coil current is permanently equal to the drive current $I$. The experiment consists of two working phases:

1. In a "flux-up" mode, the Josephson device irradiated by microwaves at frequency $f$ is biased on the $n=1$ Shapiro step for a time interval $t$. Consequently, the flux in the coil is increased by a known amount $\Delta \Phi=f \Phi_{0} t$ since the emf of the superconducting coil is maintained equal to the Josephson voltage during the process.

2. Then the Josephson device is biased on the $n=0$ step to keep $\Phi$ constant so that the floated body reaches an equilibrium position. The vertical position of the center of gravity of the body is then measured by laser interferometry while the drive current is measured by recording the voltage drop $V$ across a calibrated resistor $R$.

The energy supplied by the external source between any two equilibrium positions at heights $z_{\mathrm{L}}$ and $z_{\mathrm{H}}$, with subscripts $\mathrm{L}$ and $\mathrm{H}$ corresponding to low and high positions respectively, is

$$
\int_{\Phi_{\mathrm{L}}}^{\Phi_{\mathrm{H}}} I \mathrm{~d} \Phi=\frac{1}{2}\left(\Phi_{\mathrm{H}} I_{\mathrm{H}}-\Phi_{\mathrm{L}} I_{\mathrm{L}}\right)+m g\left(z_{\mathrm{H}}-z_{\mathrm{L}}\right) .
$$

The first term on the right-hand side corresponds to the increase of the magnetic energy of the coil-floating body system, and the second term is the work provided by the magnetic field to increase the gravitational energy of the body.

3) The experiments using the Watt balance or the floating body method lead to the determination of the Planck constant in terms of the kilogram with the double assumption that $2 e / h$ and $h / e^{2}$ are the true constants involved in the acJE and QHE.

4) NMIJ is the National Metrology Institute of Japan. 


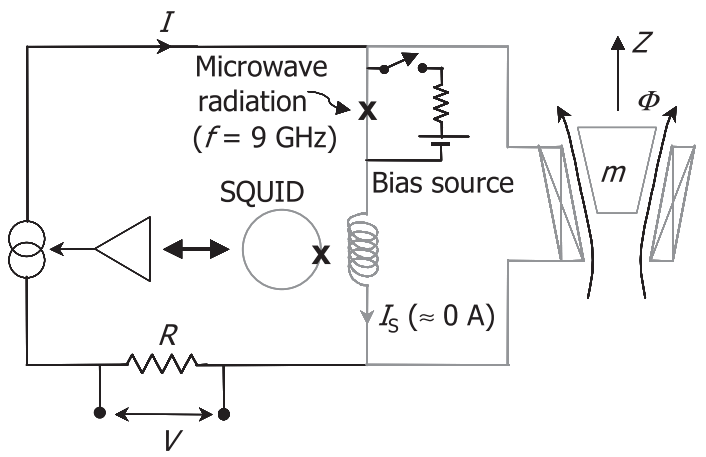

(a)

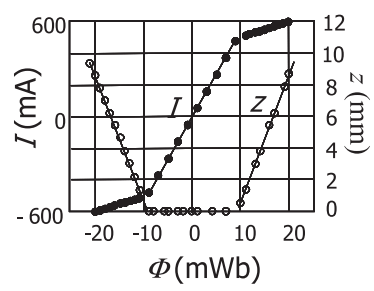

(b)

Fig. 9.2 Principle of the floating superconductor body method for the determination of $\Phi_{0}$ in SI units. (a) Schematic of the experimental set-up at NMIJ. The superconducting part of the circuit is in gray. (b) Equilibrium trajectory: coil current $I$ and vertical displacement $z$ as functions of the magnetic flux $\Phi$ induced by the coil.

The process of repeating successively the "flux up" and "constant flux" modes provides a set of data $(I, z)$ as a function of $\Phi$ which describe a so-called equilibrium trajectory [10], as shown in Figure 9.2 (b). The trajectory data allow one to calculate an SI value of $\Phi_{0}$ and thus $2 e / h$ or $K_{\mathrm{J}}$. From the energy Eq. (9.1), a value of $\Phi_{0}{ }^{2}$ is indeed deduced in terms of kilogram, meter and second since both the flux change and the current can be expressed in units of $\Phi_{0}$.

The main obstacles to reducing the uncertainty level to well below one part in $10^{6}$ presently reached at NMIJ reside in unwanted energy losses, insufficient understanding of mass metrology in the low temperature environment, and imperfect diamagnetism. The uncertainty component related to null current detection is estimated to be of the order of one part in $10^{7}$ with a SQUID ampermeter whose current sensitivity has been adjusted to $110 \mu \mathrm{A} / \Phi_{0}[11]$.

\subsection{3}

\section{Other Precise SQUID-Based Voltage Measurements}

SQUID picovoltmeters have been used in precise comparisons of low-resistancevalue standard resistors in standards laboratory applications but are generally only useful where cryogenic resistors are involved $\left(R_{\mathrm{i}}\right.$ in Figure 9.1) [14]. For comparing resistance standards of $100 \Omega$ at room temperature or quantum Hall resistance standards operating on the $i=2$ or $i=4$ plateau (12906 $\Omega$ and $6453 \Omega$, respectively) at very low temperature $(T<1 \mathrm{~K})$, it is important to develop SQUIDbased null detectors with a noise equivalent resistance $\left(R_{\mathrm{N}}\right)$ at $300 \mathrm{~K}$ less than that of the best nanovoltmeter commercially available $\left(R_{\mathrm{N}} \approx 20 \Omega\right)$. The SQUID has thus been used as a null detector in a CCC-based resistance bridge in which a $100-\Omega$ resistance standard placed at room temperature was calibrated against quantum Hall resistance standards operating on the $i=2$ or $i=4$ plateau at $1.3 \mathrm{~K}$ with an uncertainty of a few parts in $10^{9}[15]$. 
Picovoltmeters have also been fabricated using cuprate high-temperature superconductor (HTS) SQUIDs. Miklich et al. fabricated a YBCO SQUID with a YBCO input coil which demonstrated a $2.3 \mathrm{pV} / \mathrm{Hz}^{1 / 2}$ sensitivity for signals at $1 \mathrm{~Hz}$ [16]. Eriksson et al. were able to demonstrate a possible application of an HTS SQUID picovoltmeter as a means of testing for transients on high-voltage power supply transmission lines, with a speed of response which was superior to conventional methods [17].

The early work on the CCC described in the next section was aimed at Josephson voltage measurements, using cryogenic resistors to scale the voltage to higher levels $[18,19]$. In the 1970 s, the primary voltage standards were indeed based on single overdamped Josephson junctions which generated a voltage of only a few millivolts (typically working on high-order steps, $n>500$ at frequency $f \approx 10 \mathrm{GHz}$ ). By means of this cryogenic voltage comparator, it was possible to calibrate secondary standards such as the well-known 1.018-V saturated Weston cells (still in use nowadays) with an uncertainty of $10 \mathrm{nV}, 10$ times lower than the uncertainty obtained with the method employing voltage dividers.

\section{3}

\section{Cryogenic Current Comparator (CCC)}

\subsection{1}

\section{Principle of the CCC}

The CCC was invented by Harvey in 1972 [20]. The principle rests on Ampère's law and perfect diamagnetism of the superconductor in the Meissner state. Consider two wires inserted into a superconducting tube of wall thickness several times larger than the London penetration depth (Figure 9.3). Currents $I_{1}$ and $I_{2}$ circulating through these wires will induce a supercurrent $I$ flowing up the inner surface of the tube and back down the outer surface in such a way that a null magnetic flux density is maintained inside the tube. By applying Ampère's law to a contour in the bulk, labelled a in Figure 9.3, we find that

$$
\oint_{\mathrm{a}} B \mathrm{~d} l=0=\mu_{0}\left(I_{1}+I_{2}-I\right)
$$

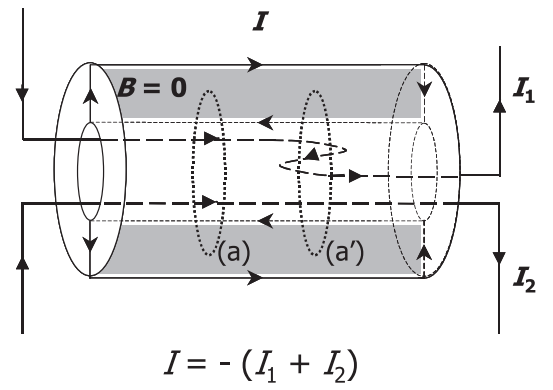

Fig. 9.3 Principle of a CCC. 
which yields the equality of the currents:

$$
I=I_{1}+I_{2} .
$$

If the wires carrying $I_{1}$ and $I_{2}$ pass through the tube $N_{1}$ and $N_{2}$ times respectively, the supercurrent $I$ is equal to

$$
I=N_{1} I_{1}+N_{2} I_{2}
$$

These equalities are valid independent of the position of the wires inside the tube, even if the wires describe some " $\mathrm{S}$ " path (Figure 9.3, case $\mathrm{a}^{\prime}$ ). Only the current density distribution on the inner surface of the tube can be inhomogeneous in the case of asymmetric positioning of the wires, whereas the current density distribution on the outer surface stays homogeneous. Here is the key reason for the high level of precision of the CCC.

In the real case of a tube of finite length, the equalities above are in fact altered by the unscreened magnetic flux induced by the currents $I_{1}$ and $I_{2}$ at the ends. To overcome these end effects and at the same time to make the device practical with a limited size, the tube is shaped in a torus with its ends being overlapped (but electrically insulated) like a "snake swallowing its tail" (Figure 9.4). The longer is the overlap, the more efficient is the screening of the magnetic flux which tends to leak through the gap of the overlap. This is the method proposed by Sullivan and Dziuba for improving the exactness of the CCC current ratio [21]. Currents $I_{1}$ and $I_{2}$ of opposite direction flowing in two windings of number of turns $N_{1}$ and $N_{2}$ induce a supercurrent $I=N_{1} I_{1}-N_{2} I_{2}$. The external magnetic flux $\Phi$, which results only from the supercurrent, is detected by a SQUID through a flux transformer composed of a pickup coil wound very close to the toroidal shield (on its inner or outer surface) and the input coil of the SQUID. The output voltage of the SQUID is then converted to a current which feeds back one of the two windings

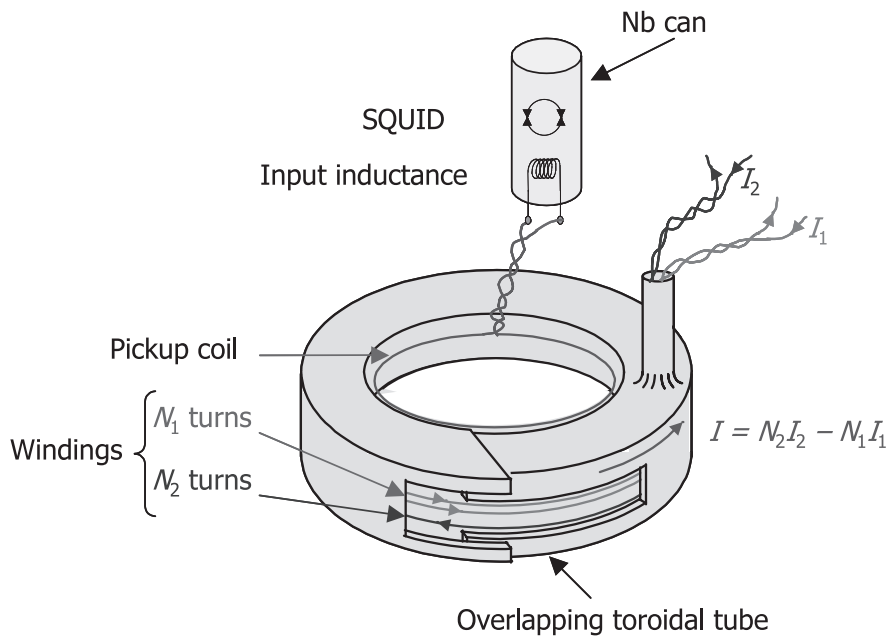

Fig. 9.4 Schematic of a type I CCC. 
to null the magnetomotive forces. From this ampere turn balance results the equality of the ratios:

$$
I_{1} / I_{2}=N_{2} / N_{1}
$$

A second type (II) of CCC, proposed by Grohmann and coworkers in 1973, can also be constructed by placing the pickup coil inside the toroidal superconducting shield and by wrapping the windings outside, like a conventional transformer with windings around the ferromagnetic torus [22]. The superconducting shield also overlaps itself around the torus. Figure 9.5 shows an example of a type II CCC which is presently used in metrology and is described in Section 9.3.5 [23]. Note that in this configuration, the SQUID could be placed inside a superconducting shield and thus become completely immune to parasitic magnetic flux.

All CCCs currently used in NMIs are based on low-temperature superconducting (LTS) materials, particularly lead for the overlapping shield, because these materials guarantee high current resolution (reduced Johnson noise due to low working temperature, high level of screening against unwanted magnetic fields), and high accuracy (because their high critical current density allows a complete Meissner effect in the bulk). However investigations have been carried on HTSbased CCCs and some promising results have been reported [24-28]. Different geometries have been designed depending on the particular application. For instance, Early et al. have been able to fabricate a split-toroid YBCO CCC which could be suitable for resistance ratio or low current measurements [28]. Another example, which is described in Section 9.3.6, is a CCC made of a YBCO-coated tube. It has been developed at the National Physical Laboratory (NPL) for measuring charged particle beams.

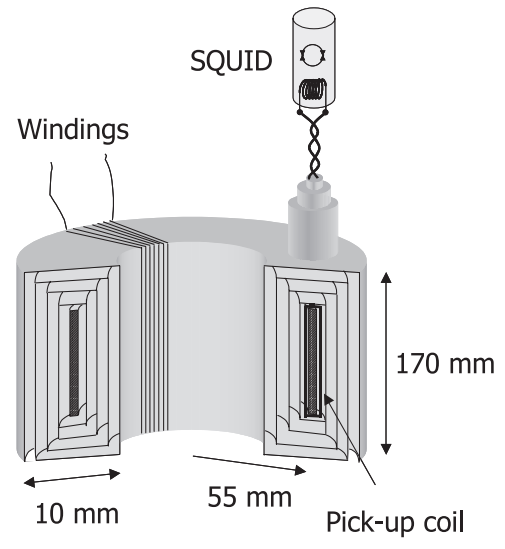

Fig. 9.5 Schematic of a type II CCC. 


\subsection{2}

\section{Limits to Accuracy of CCC Ratio Measurements}

\subsubsection{Sensitivity and Current Resolution}

The performance of the CCC in terms of sensitivity and noise depends strongly on the flux transformer (FT), which has to match the SQUID to the overlapping toroidal shield as well as possible, as shown schematically in Figure 9.6.

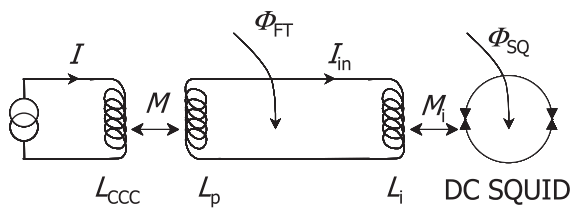

Fig. 9.6 Basic flux transformer (FT) circuit.

\section{Sensitivity of the CCC}

The sensitivity of the CCC, $S_{\mathrm{CCC}}$, is defined as the variation of the supercurrent $I$ circulating on the surface of the overlapping tube which creates a magnetic flux variation $\Phi_{\mathrm{SQ}}$ of one $\Phi_{0}$ across the SQUID ring. In ampereturn $/ \Phi_{0}$

$$
S_{\mathrm{CCC}}=\left|\partial \Phi_{\mathrm{SQ}} / \partial I\right|^{-1}=\left|\partial I / \partial I_{\text {in }}\right| / M_{\mathrm{i}}
$$

where $M_{\mathrm{i}}$ is the mutual inductance of the SQUID loop and input coil and $I_{\text {in }}$ is the circulating current in the flux transformer. Applying conservation of magnetic flux to the superconducting flux transformer, assuming zero initial flux, $\Phi_{\mathrm{FT}}=$ $\left(L_{\mathrm{p}}+L_{\mathrm{i}}\right) I_{\mathrm{in}}+M I=0$, gives

$$
S_{\mathrm{CCC}}=\left(L_{\mathrm{p}}+L_{\mathrm{i}}\right) / M M_{\mathrm{i}}
$$

where $L_{\mathrm{p}}$ is the inductance of the pickup coil, $L_{\mathrm{i}}$ is the input inductance of the SQUID and $M$ is the mutual inductance between the overlapping toroidal tube and the pickup coil. It is easy to show that the sensitivity reaches the optimal value

$$
S_{\mathrm{CCC}}^{\mathrm{opt}}=(2 / k)\left(L_{\mathrm{i}} / L_{\mathrm{CCC}}\right)^{1 / 2} / M_{\mathrm{i}}
$$

where $L_{\mathrm{CCC}}$ is the inductance of the overlapping toroidal tube, for a number of turns of the pickup coil

$$
N^{\text {opt }}=\left(L_{\mathrm{i}} / l_{\mathrm{p}}\right)^{1 / 2} .
$$

Here $k$ is the coupling constant between the pickup coil and the overlapping shield $\left(M=k\left(L_{\mathrm{p}} L_{\mathrm{CCC}}\right)^{1 / 2}\right)$ and $l_{\mathrm{p}}$ is the inductance corresponding to a single turn of the pickup coil $\left(L_{\mathrm{p}}=N^{2} l_{\mathrm{p}}\right)$. 
In practice, the overall CCC and SQUID ${ }^{5}$ system is surrounded by (at least) one external superconducting shield to obtain an environmental magnetic field weak enough and essentially very stable. Consequently the inductive coupling between this external shield and both the overlapping shield and the pickup coil has to be taken into account to calculate $N^{\text {opt }}$ and $S_{\text {CCC }}^{\text {opt }}$ and Eq. (9.9) is no longer valid ${ }^{6}$.

Several numerical methods have been proposed in the literature to calculate $S_{\text {CCC }}$ of type I CCCs and good agreement has been generally found between calculated and measured values [29-31]. From the method proposed by Sesé and coworkers analytical formulae for $S_{\mathrm{CCC}}$ can be derived, provided some realistic conditions are fulfilled [32,33]. This method, based on a finite element calculation, takes into account not only the influence of the external shield but also the mirror effect of the overlapping toroidal shield on the pickup coil. The perpendicular magnetic field induced by the coil on the wall of the shield is cancelled by the superconducting material, like an image coil which generates an opposite magnetic field. This effect results in an effective inductance $L_{\mathrm{p}}{ }^{\prime}$ and a mutual inductance $M$ given by the relations:

$$
\begin{aligned}
& L_{\mathrm{p}}{ }^{\prime}=\left(1-k^{\prime}\right) L_{\mathrm{p}}+k N^{2} L_{\mathrm{CCC}}^{\prime}, \\
& M=k N L_{\mathrm{CCC}}^{\prime},
\end{aligned}
$$

provided $k^{\prime}$, the coupling constant between the pickup coil and its image, is close to 1 [32]. Here $k$ is a coupling parameter between the pickup coil and the overlapping toroidal shield characterized by an effective inductance $L_{\text {CCC }}^{\prime}$ In the ideal case where $k^{\prime}=1$, the optimal number of turns $N^{\text {opt }}$ and the optimal sensitivity $S_{\mathrm{CCC}}^{\mathrm{opt}}$ are given by

$$
N^{\mathrm{opt}}=\left(L_{\mathrm{i}} / L_{\mathrm{CCC}}^{\prime}\right)^{1 / 2}
$$

and

$$
S_{\mathrm{CCC}}^{\mathrm{opt}}=(2 / k)\left(L_{\mathrm{i}} / L_{\mathrm{CCC}}^{\prime}\right)^{1 / 2} / M_{\mathrm{i}}=(2 / k) N^{\mathrm{opt}} / M_{\mathrm{i}}
$$

In practice, Eq. (9.12) combined with the calculated value of the effective inductance $L_{\text {CCC }}^{\prime}$ provide a good estimate to the designer of the number of turns required on the pickup coil. Note that, for Eqs. (9.12) and (9.13) to be valid, $L_{\mathrm{i}}$ has to be larger than $L_{\mathrm{CCC}}^{\prime}$ which is often the case.

5) In general, the SQUID itself is inserted inside a superconducting shield (typically a $\mathrm{Nb}$ can) as shown in Figure 9.3.

6) If the SQUID is mounted very close to the overlapping tube, the influence of the super- conducting shield of the SQUID on the calculation of $S_{\mathrm{CCC}}$ would also have to be taken into account: an example is a $\mathrm{Nb}$ can which largely occupies the central zone of the torus (Figure 9.4). 


\section{Current Resolution}

The most relevant characteristic of the CCC is its current resolution $\delta I$ in terms of $\mathrm{A} / \mathrm{Hz}^{1 / 2}$ and is defined as the square root of the power spectral density of current noise referred to the CCC input, or equivalently as the minimum measurable supercurrent circulating per $\mathrm{Hz}^{1 / 2}$ in the overlapping tube of the CCC. The complete expression for $\delta I$ is given by the relation

$$
\delta I=\left[4 k_{\mathrm{B}} T / R_{\mathrm{in}}+8 \varepsilon / N_{1}^{2} k^{2} L_{\mathrm{CCC}}^{\prime}+\left(S_{\Phi \mathrm{ext}} / N_{1} L_{\mathrm{CCC}}^{\prime}\right)^{2}\right]^{1 / 2}
$$

where $N_{1}$ is the number of turns of the primary winding of the CCC. The first term corresponds to the Johnson noise of the input resistor at temperature T. The second term is the contribution of the SQUID with an energy resolution $\varepsilon$ when the optimal sensitivity of the CCC is reached. The third term comes from the external magnetic flux noise with a power spectral density $S_{\text {कext }}$ [34]. This last term becomes negligible with careful shielding as described below. The dominant noise arises from one of the two first terms, depending on the CCC application. When a CCC is used for comparing resistance standards (Section 9.3.3), the Johnson noise they deliver cannot be avoided and consequently the number of turns of the primary winding is increased to a limiting value (typically around 2000) above which the noise contribution of the SQUID becomes negligible. For low current measurements (Section 9.3.4), where high input resistances are involved, only the SQUID noise contributes.

The current resolution of a CCC fitted with a high-permeability toroidal core is given by a relation which slightly differs from Eq. (9.14), in which $L_{\mathrm{CCC}}^{\prime}$ will be replaced by another effective inductance including the permeability of the ferromagnetic core. The power spectral density of the current noise of the core itself, $\left(\delta I_{\mathrm{C}}\right)^{2}$, obviously has to be added.

\section{Some Details on Design}

From the reported results of various calculations and measurements of the effective inductances, $L_{\mathrm{CCC}}^{\prime}$ and $L_{\mathrm{p}}^{\prime}$, and of the sensitivity and current resolution a preliminary design for a type I CCC can be made as follows [29-37].

The overlapping toroidal shield has to be designed with a mean radius $a$ as high as possible and a section $h \times w$ (see Figure 9.7) as small as possible since a maximum inductance is required. These requirements are evident from the relation below, which gives $L_{\mathrm{CCC}}$ without taking into account the surrounding external shield

$$
L_{\mathrm{CCC}}=\mu_{0} a[\ln (8 a / r)-2]
$$

where $r=(w h / \pi)^{1 / 2}$ is the radius of a circular section whose area is equal to that of the rectangular section of the toroidal shield (Figure 9.7). In fact, this relation has been found to be in good agreement, to within a few per cent, of the value found by numerical calculations of the distribution of the current circulating on the surface of the overlapping shield [36]. 


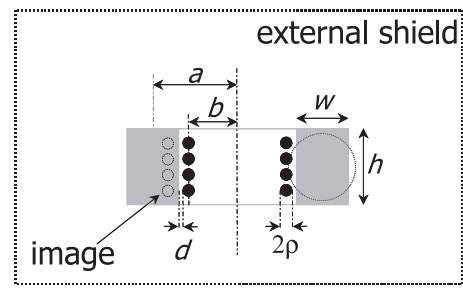

Fig. 9.7 Parameters of a toroidal shield and pickup coil for a type I CCC.

However, these two parameters are fixed by the experimental constraints, the section $h \times w$ by the total number of turns of the winding and the mean radius $a$ by the radius of the external shield, which is itself limited by the size of the cryostat neck.

By considering the system as a magnetic circuit and introducing the relevant reluctances, Sesé et al. have proposed a simple rule to optimize to a good approximation the size of the overlapping shield with respect to the external shield [37]. They find that $L_{\mathrm{CCC}}$ is maximized when the internal area $A_{\text {int }}\left(=\pi b^{2}\right)$ enclosed by the torus is equal to the external area between the torus and the external shield $A_{\text {ext }}\left(=\pi R^{2}\right.$ shield $\left.-\pi(b+w)^{2}\right)$, as illustrated in Figure 9.8. This result follows from the equality of the reluctances $\Re_{1}=h /\left(\mu_{0} A_{\text {int }}\right)$ and $\Re_{2}=h /\left(\mu_{0} A_{\text {ext }}\right)$, with $A_{\text {int }}+A_{\text {ext }}$ kept constant.

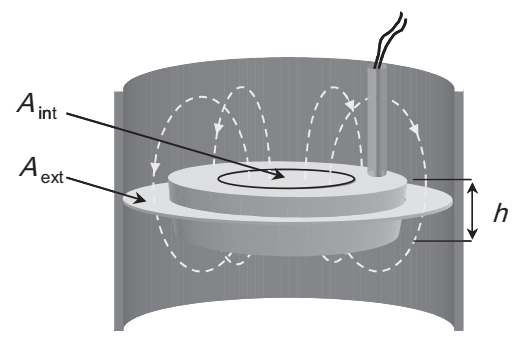

Fig. 9.8 Effect of external shield on type I CCC.

The optimal value of the mean radius of the toroidal shield is given by

$$
a^{\text {opt }}=\left[\left(R_{\text {shield }}^{2}-w^{2} / 2\right) / 2\right]^{1 / 2} .
$$

It is noteworthy that the mean radius a could be made larger if the axis of the toroidal shield is made perpendicular to the external shield axis.

The pickup coil has to be wound as close as possible to the inner or outer surface of the overlapping shield $(d / a \ll 1$, see Figure 9.7) and the turns have to be suitably spaced, as described below. The coupling between the overlapping shield and the pickup coil is indeed much improved by minimizing the coupling of each turn to the rest of the turns, each turn being coupled only to its own image [32]. Several possibilities have been proposed: individually shielding the turns by means of superconducting sheath, placing the wire inside a superconducting tube 


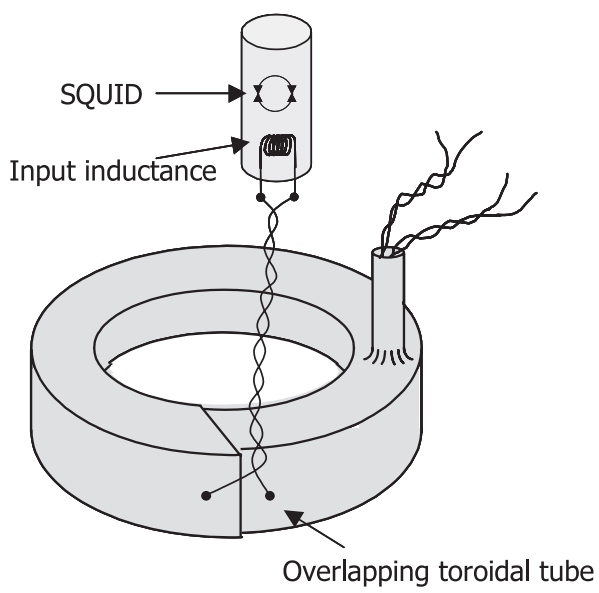

Fig. 9.9 Overlapping toroidal shield used as a pickup coil.

or using a wide tape instead of a wire [33]. Another way is to use a SQUID with an input inductance $L_{\mathrm{i}}=L_{\mathrm{CCC}}^{\prime}$, equivalent to that of a single-turn pickup coil. A perfect matching has thus been achieved by connecting the input coil of the SQUID directly to the overlapping shield as shown on Figure 9.9 [38].

For a good current resolution the SQUID has to be chosen with high energy resolution, but without a severe restriction on the input inductance (provided that $L_{\mathrm{i}} \geq L_{\text {CCC }}^{\prime}$ ) since this inductance has no influence on $\delta I$.

Good current resolution strongly depends on the screening of environmental magnetic fields by additional external shields. The shields are used more for obtaining high stability of the residual magnetic field rather than for reducing the latter down to a non measurable level. The condition to be fulfilled is $S_{\Phi \text { ext }}^{1 / 2}$ $<10^{-4} \Phi_{0} / \mathrm{Hz}^{1 / 2}$, given the typical values $\varepsilon=10^{-31} \mathrm{~J} / \mathrm{Hz}$ (in the white noise regime) and $L_{\mathrm{CCC}}^{\prime}=10 \mathrm{nH}$ in Eq. (9.14). In terms of magnetic flux density, the condition becomes $S_{\text {Bext }} 1 / 2<0.3 \mathrm{fT} / \mathrm{Hz}^{1 / 2}$ for a typical CCC with a $20-\mathrm{mm}$ inner diameter. Here, $S_{\Phi \text { ext }}$ and $S_{\text {Bext }}$ are the spectral densities of the external magnetic flux and field noise. Consequently, the total attenuation required for screening a parasitic magnetic field like that of the Earth's $\left(\left|B_{\text {Earth }}\right| \approx 50 \mu \mathrm{T}\right)$ should exceed $220 \mathrm{~dB}$. This value can then be considered as an upper limit value for obtaining a highly stable magnetic environment.

\subsubsection{Exactness of the CCC Current Ratio}

\section{Uncertainty of the Winding Ratio}

A high accuracy of current ratio measurement is reached when the superconducting tube covering the windings overlaps itself over a length $l$ large enough to minimize the flux $\Phi_{1}$ leaking through the gap of the overlap. This leakage flux carries the information on the position of the windings. Theoretical error values of the order of one part in $10^{10}$ or less have been reported for both types of CCC [39]. 
The relationship below, which is a simplified form of the relation proposed by Seppä for a type I CCC, gives the maximum error in terms of magnetic flux:

$$
\Phi_{1} / \Phi=[\delta \exp (-l / r)][a \ln (a / r)]^{-1}
$$

where $\Phi$ is the total magnetic flux, $\delta$ is the gap thickness, $r$ is the equivalent radius of the tube $\left(r=(w h / \pi)^{1 / 2}\right.$ with the same notations as in Figure 9.7) and $a$ is the mean radius of the torus [40].

For a one-turn overlap of the shield $(l=2 \pi a)$, with typical values $a=15 \mathrm{~mm}, r=$ $3 \mathrm{~mm}$ and maximum value $\delta=1 \mathrm{~mm}$, the error is around $10^{-16}$, smaller than the least measurable values which have been reported to be $10^{-11}$.

Note that a possible error on the winding ratio could exist due to the natural imperfection of the ends of each winding, which may form kinks. In practice, this error is nulled first by twisting the output wires and then, in the case of a type I CCC, by inserting the output wires in the superconducting coaxial tubes forming a chimney, such as that shown in Figure 9.4, for example. The resulting magnetic flux due to the winding ends is indeed strongly screened by the superconducting tube, the efficiency increasing as a function of the height/diameter ratio of the tube.

In practice, a CCC is generally made of a series of windings with numbers of turns in an arithmetic progression and two identical windings (for instance 15, $15,30,60,120, \ldots)$. This enables one to test the efficiency of the superconducting toroidal shield by a self-calibration of the ratio 1:1. A current passing through two windings with the same number of turns connected in series-opposition can induce an ampere-turn imbalance, which gives the ratio error [41]. Superconducting wires are generally used for the CCC windings in order to make static leakage currents negligible and to reduce to a few ohms the resistance of links between current sources and resistances to be compared.

\section{Frequency Effect}

Most of the measurements involving CCCs are carried out at ultralow frequencies between $10 \mathrm{mHz}$ and $0.5 \mathrm{~Hz}$ with the expectation that the results will not be altered by any frequency effect. A few measurements carried out at frequencies from $1 \mathrm{~Hz}$ to $10 \mathrm{kHz}$ produced interesting results and enable one to establish an upper limit on the error in the current ratio and to reduce it. According to Grohmann et al., the error sources arise from various capacitances inside the CCC (shunting capacitance between the leads, stray capacitance between ground and leads, shunting capacitances between the turns of the winding and stray capacitance between ground and winding) [42]. They may partly cancel each other out due to opposite signs. For example, consider two windings shown schematically in Figure 9.10. The true value of the current crossing the winding labelled 1 is given by

$$
I_{1 \mathrm{~T}}=I_{1}-I_{1}{ }^{\prime}+I_{2}{ }^{\prime \prime},
$$




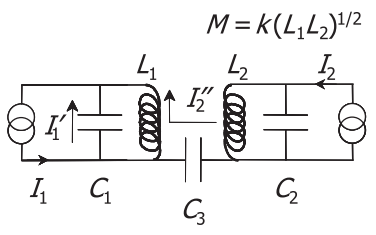

Fig. 9.10 Schematic of two windings with leakage currents crossing the shunt capacitances $C_{1}$ and $C_{3}$.

where $I_{1}{ }^{\prime}$ denotes the leakage oscillating current crossing the shunt capacitance of the winding and $I_{2}{ }^{\prime \prime}$ corresponds to the oscillating current leaking through the capacitance between the two windings. It seems possible to cancel the errors, at least partly, by means of coaxial windings for type II CCCs or by placing a supplementary capacitor in parallel to the winding or between one of the winding terminals and the ground for type I CCCs [43]. The type II geometry is the most suited for ac applications of CCC. Errors reduced to one part in $10^{8}$ at $10 \mathrm{kHz}$ have been reported [39].

For a type I CCC, a simple approximate expression for the error on the current ratio $I_{1} / I_{2}$ can be given, in terms of the self-inductance of the windings $L_{1}$ and $L_{2}$ and their mutual inductance $M=k\left(L_{1} L_{2}\right)^{1 / 2}$ with a coupling factor $k$

$$
\delta\left(I_{1} / I_{2}\right) /\left(I_{1} / I_{2}\right)=(1-k) L_{1} C \omega^{2}
$$

for the case of large ratio $I_{1} / I_{2} \gg 1$ (i.e. $L_{1} \gg L_{2}$ ). Here $C$ denotes the leakage capacitance (shunt or stray) and $\omega$ is the frequency [44]. The term (1-k) $L_{1}$ can be deduced from the measurement of the emf across winding 1 induced by an alternating current passing through it. The measurement of the capacitance between winding 1 on one side and winding 2 and ground on the other leads to an approximate value of $C$. Another possible way to estimate the ratio error is via the determination of the lowest resonance frequency of the $\operatorname{CCC} f_{\text {res }}$ given by

$$
\delta\left(I_{1} / I_{2}\right) /\left(I_{1} / I_{2}\right)=(1-k)\left(f / f_{\text {res }}\right)^{2} .
$$

We have assumed that each winding combined with its leakage capacitance behaves as an LC tank circuit, where the lowest resonance frequency is $f_{\text {res }} \approx$ $1 / 2 \pi L_{1} C$ (if $L_{1} \gg L_{2}$ ). The resonant peaks can easily be observed on the current (or flux) noise spectrum recorded at the system output [35, 45-47].

\section{Finite Open Loop Feedback Gain}

An uncertainty in the value of the current ratio measured by the CCC arises from the finite open loop gain of the SQUID operating in a flux-locked loop mode. The error in current ratio can be easily deduced from the simplified block diagram shown in Figure 9.11. Currents $I_{1}$ and $I_{2}$ applied to the CCC through its (primary) winding of $N_{1}$ turns and (secondary) winding of $N_{2}$ turns respectively, induce a magnetic flux difference $\delta \Phi=\Phi_{1}-\Phi_{2}$ at the SQUID. The loop gain of the CCC, $G_{\mathrm{L}}$, which corresponds to the ratio $\Phi_{1} / \delta \Phi \approx \Phi_{2} / \delta \Phi$, is given by

$$
G_{\mathrm{L}}=N_{2} G_{\text {Electronics }} V_{\Phi} / R_{\mathrm{f}} S_{\mathrm{CCC}}
$$




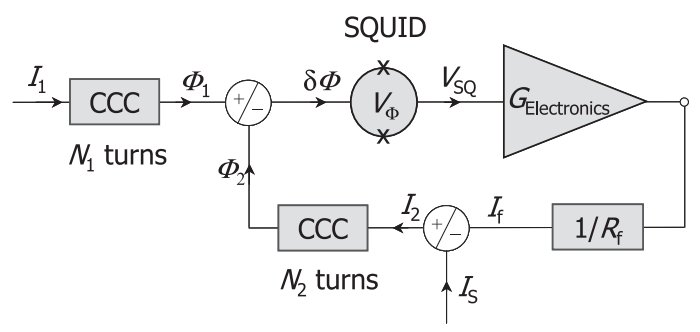

Fig. 9.11 CCC block diagram.

where $G_{\text {Electronics }}$ is the gain of the feedback electronics (see Chapter 4), $V_{\Phi}$ is the optimized transfer coefficient of the SQUID and $R_{\mathrm{f}}$ is the feedback resistance.

First, consider the case of current $I_{2}$ only delivered by the SQUID feedback electronics. The error in current ratio is simply equal to the reverse of the loop gain,

$$
I_{2}=I_{\mathrm{f}}=\left(N_{1} / N_{2}\right) I_{1}\left(1-1 / G_{\mathrm{L}}\right) \text {. }
$$

Typically, $G_{\mathrm{L}}$ reaches $100 \mathrm{~dB}$ at a frequency of $10 \mathrm{mHz}$ for a bandwidth of $1 \mathrm{kHz}$ (in dynamic mode, $G_{\mathrm{L}}(f) \propto f_{\text {cut-off }} / f$ ), and so induces an error of one part in $10^{5}$. Second, consider the case of current $I_{\mathrm{S}}$ delivered by a secondary current source which is servo-controlled by the primary source in such a way that $I_{\mathrm{S}} / I_{1}$ is preliminarily adjusted: $I_{\mathrm{S}} / I_{1}=N_{1} / N_{2}(1 \pm x)$. The sum of currents circulating through the secondary winding is thus given by

$$
I_{2}=\left(N_{1} / N_{2}\right) I_{1}\left(1 \pm x / G_{\mathrm{L}}\right) \text {. }
$$

The error $x / G_{\mathrm{L}}$ becomes insignificant if $x<10^{-5}$, an adjustment value not so difficult to achieve. The error decreases if $N_{2}$ increases but the stability margin decreases as well.

\subsection{3}

\section{Resistance Ratio Measurement}

\subsubsection{Introductory}

The resistance bridges currently used in NMIs to calibrate material resistance standards (wire-wound resistors) against quantum Hall resistance standards (QHRS) are based on the type I CCC and generally this CCC is coupled to a commercial rf or dc SQUID. Precise resistance ratio measurement is made possible via the current ratio measurement made with a very high accuracy. The CCC presents two advantages compared to the conventional direct current comparators, commercially available and operating with ferromagnetic cores:

- The resolution is markedly improved, by a factor of 100 at least. For a $\mathrm{CCC}$, the square root of the noise power spectral density in terms of ampereturns is typically between $10^{-9}$ and $10^{-10}$ ampereturn/ $\mathrm{Hz}^{1 / 2}$ in the white noise region, or of the order of $100 \mathrm{fA} / \mathrm{Hz}^{1 / 2}$ for the largest windings. This low noise level is mainly due to the SQUID used as magnetic flux detector. 
- The uncertainty in current ratio does not exceed one part in $10^{9}$ (more often than not, it is reduced down to one part in $10^{10}$ ) compared to a few parts in $10^{8}$ with a ferromagnetic-core-based direct current comparator ${ }^{7)}$ operating at room temperature.

With such properties, the CCC allows one to demonstrate the universality of the von Klitzing constant $R_{\mathrm{K}}$ (corresponding to $h / e^{2}$ ) derived from the QHE through the relation $R_{\mathrm{H}}(i)=R_{\mathrm{K}} / i$ where $R_{\mathrm{H}}$ is the quantum Hall resistance and $i$ is an integer corresponding to the Hall quantum plateau number (see $[49,50]$ and references therein). Under some experimental conditions and following specific technical guidelines [51], the independence of $R_{\mathrm{K}}$ on QHE device characteristics (type, materials, channel width, contacts), the number $i$ and experimental parameters (temperature, measuring current, magnetic field) has been demonstrated at levels down to a few parts in $10^{10}[52,53]$.

Moreover, on-site bilateral comparisons of complete QHE systems carried out between the Bureau International des Poids et Mesures (BIPM) and some NMIs during the past decades [54-58] or more recent comparisons via $1 \Omega$ or $100 \Omega$ travelling standards have shown excellent agreement of a few parts in $10^{9}[59,60]$. These results, which have to be ascribed to the use of CCC bridges in such comparisons, strongly support the universality of $R_{\mathrm{K}}{ }^{8}$.

Typically, the secondary resistance standards which are calibrated against QHRS have nominal values of $1 \Omega$ and $10 \mathrm{k} \Omega$. They are used as reference standards for routine calibration purposes. Their values are rather far from that of $R_{\mathrm{K}} / 4$ and $R_{\mathrm{K}} / 2$ (around $6453 \Omega$ and $12906 \Omega$, respectively) quantum values which are the most often used with single QHE devices. Consequently, their calibration against QHRS has to be carried out in several steps. In contrast, for example, to the method involving resistance build-up networks, the CCC bridge described below makes the calibration process of these secondary standards more simple, restricted to only two steps, by means of transfer standards such as $100 \Omega$ and $1 \mathrm{k} \Omega$ resistance standards.

Note that some NMIs have developed specific CCC bridges to cover a more extended measurement range, up to $100 \mathrm{M} \Omega$ via a direct comparison with QHR $[47,62]$, and even up to $1 \mathrm{G} \Omega[63]^{9}$.

7) In the best case, measurement uncertainties of the order of one part in $10^{9}$ have been reported with a non-commercial room-temperature current comparator working at $1 \mathrm{~Hz}$ [48].

8) That confirms the validity of the international recommendation in 1988 for the use of the quantum Hall resistance as the primary resistance standard, too. As for the ac Josephson effect (see footnote 1), CIPM has defined the von Klitzing constant $R_{\mathrm{K}}$ as an estimate of $h / e^{2}$ and for calibration purposes has assigned to it one single value $R_{\mathrm{K}-90}=$
$25812.807 \Omega$ [61]. Note that the uncertainty on $R_{\mathrm{K}}$ presently given by the CIPM is 1 part in $10^{7}$ in terms of SI units.

9) With the same aim of scaling the resistance in terms of $R_{\mathrm{K}}$ over a wide range, quantum Hall array resistance standards (QHARS) with nominal quantum values from $100 \Omega$ to $1.29 \mathrm{M} \Omega\left(50 R_{\mathrm{K}}\right)$ are currently developed. The arrays are composed of a large number of single Hall bars connected in series or in parallel with multiple links (see for instance $[64,65]$ and references therein). 


\subsubsection{Resistance Bridge Based on Type I CCC}

The CCC is connected to a double constant current source which feeds resistors of resistances $R_{1}$ and $R_{2}$ to be compared, with primary and secondary currents $I_{1}$ and $I_{2}$ respectively, as shown in Figure 9.12. Here, the secondary current source is slaved on the primary current source in such a way that the current ratio can be reliably adjusted allowing the SQUID to be properly and accurately flux locked, as discussed in Section 9.3.2.2 [41, 49].

In the ideal case, the operation should consist of setting the current ratio to equal the voltage drops across the resistances, i.e., to null the voltage drop through a detector connected to the low voltage terminals of the resistances: $R_{1} I_{1}=R_{2} I_{2}$. Consequently the resistance ratio $R_{1} / R_{2}$ will be equal to the winding ratio $N_{1} / N_{2}$.

In real cases, a fraction $\varepsilon$ of the current $I_{2}$ has to be diverted to an auxiliary winding of $N_{\mathrm{a}}$ number of turns to balance the bridge both in voltage and in ampereturns:

$$
\begin{aligned}
& N_{1} I_{1}-N_{2} I_{2}+\varepsilon N_{\mathrm{a}} I_{2}=0, \\
& R_{1} I_{1}-R_{2} I_{2}=0 .
\end{aligned}
$$

Elimination of the currents from these two relations gives the resistance ratio:

$$
\frac{R_{1}}{R_{2}}=\frac{N_{1}}{N_{2}}\left(1+\frac{N_{a}}{N_{2}} \varepsilon\right) .
$$

In the bridge illustrated in Figure 9.12, the deviation $\varepsilon$ is obtained by recording the output voltages of the null detector which correspond to the two positions of the resistive divider $\varepsilon_{ \pm}=\varepsilon \pm x$ where $x$ is typically of the order of one part in $10^{7}$. Thus $\varepsilon$ is given by

$$
\varepsilon=\left(\varepsilon_{-} V_{+}-\varepsilon_{+} V_{-}\right) /\left(V_{+}-V_{-}\right),
$$

where the voltages $V_{+}$and $V_{-}$of opposite signs correspond to $\varepsilon_{+}$and $\varepsilon_{-}$respectively [49]. This deviation $\varepsilon$ can also be obtained by using an amplifier at the output of the detector which generates a current through the auxiliary winding and a resistor placed in series. The value of $\varepsilon$ is thus deduced by measuring the voltage drop across the resistor [66]. The advantage of this second method is the ability to automate the bridge fully.

Either way, the voltages are measured by periodically reversing the current polarity in order to compensate for the unwanted thermal electromotive forces. The typical working frequency is of the order of $0.1 \mathrm{~Hz}$ or less and might be in the range where the SQUID generates $1 / f$ noise. This flicker noise may be avoided by operating the bridge at $1 \mathrm{~Hz}$ [44]. However, the current ratio has to be preadjusted both in phase and out of phase and the CCC needs a supplementary winding. Moreover, the dependence of the resistance standard on frequency has to be known. As previously mentioned, working frequencies higher than $1 \mathrm{~Hz}$ might induce significant error $\left(>10^{-9}\right)$ in the current ratio due to finite capacitive leakage and shunt between the CCC windings. 


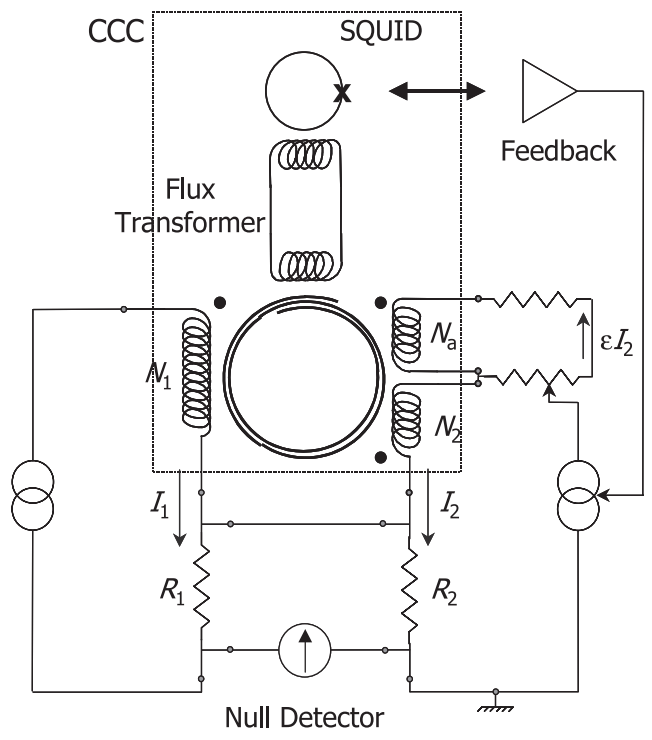

Fig. 9.12 Circuit diagram for a CCC-based resistance bridge. The toroidal shield of the CCC is symbolized by a two-turn spiral. The three dots indicate the input of coils for applying currents, which induce magnetic fields of the same polarity.

\subsubsection{Some Remarks on Construction}

The CCC has to be composed of a series of windings allowing any kind of current ratios, $1,2,4, \ldots, 100$ and particularly the ratios 64.532 and 129.064 used for measuring resistance ratios $R_{\mathrm{H}}(i) / 100 \Omega$ with $i=4$ or 2 . In the case of $R_{\mathrm{H}}(i=2) / 100 \Omega$, the numbers of turns $N_{1}$ and $N_{2}$ are usually equal to 1936 or 2065 and 15 or 16, respectively, so that the winding ratio does not deviate by more than $2 \times 10^{-5}$ from the ratio of the nominal values of resistances (12906.4035:100). Moreover, the choice of $N_{1}$ values of around 2000 is well suited to reduce the SQUID noise to a level less than the Johnson noise of the resistances to be compared.

To take full advantage of the high performance of the CCC, great care has to be taken in the construction of the bridge. For example, the bridge must have low leakage currents and good insulation between the primary and secondary circuits. The current ratio will be precisely preadjusted taking into account the finite open loop gain of the SQUID. The potentiometer must be calibrated and the nonlinearity of the nanovoltmeter used as null detector has to be measured. It is strongly recommended that the complete bridge has a unique ground point. Optocoupler isolation amplifiers are commonly used, for instance at the external feedback output of the SQUID electronics and at the output of the null detector. The IEEE transmission line through which the computer monitors the bridge and acquires data is an optical fiber. 


\section{3 .4}

Measurements of Very Low Currents from SET Sources

\subsubsection{Introduction}

By extending the concept of the sensitive current detector as proposed by Harvey in his original paper, one can use the CCC as a low-current amplifier with two characteristics never reached by any conventional device [20]. As shown below, the CCC may exhibit a current resolution around $1 \mathrm{fA} / \mathrm{Hz}^{1 / 2}$ or less over the white noise frequency range. This excellent resolution is mainly due to the low noise properties of the SQUID. The second extraordinary feature of this cryogenic amplifier is the exactness of the current gain. The metrological needs of such a CCC emerged in the 1990s when the first SET current sources, electron turnstile [67] and electron pump [68], were demonstrated and exhibited quantization of the current they deliver. These SET devices allow control of elementary charge transfer at the rate of a clock signal [69]. The amplitude of the delivered current is simply given by the product of the elementary charge $e$ and frequency $f$. Its value is rather low, of the order of $1 \mathrm{pA}$ or, at best, less than $1 \mathrm{nA}$ for more recent SET devices based on surface acoustic waves (SAW) [70]. Therefore, measurement of this current with metrological accuracy, for example within an uncertainty of one part in $10^{6}$, requires a highly accurate amplifier and the CCC is the most appropriate instrument. The development of a CCC-based current amplifier has been greatly stimulated by the demonstration of a SET-based quantum current standard (especially for subnanoampere range). The motivation is above all to close the quantum metrological triangle (QMT) (Figure 9.13) which experimentally consists of applying Ohm's law $V=R I$ directly to the voltage, resistance and current related to the ac JE, QHE and SET respectively, as first proposed by Likharev and Zorin, noting that voltage and current are both in terms of a frequency, a physical quantity which is measured with the highest accuracy nowadays [71]. Another experimental way for the closure of the QMT consists of following $Q=C V$ from the realization of a quantum capacitance standard from SET devices without, however, involving a SQUID [72, 73].

The aim of the QMT experiments is to check the consistency of the constants involved in the three quantum phenomena which are strongly believed to provide the free space values of $h / e^{2}, 2 e / h$ and $e$. In practice, the experiments determine the dimensionless product $R_{\mathrm{K}} K_{\mathrm{J}} Q_{\mathrm{X}}$, expected to be equal to 2 , where the constant $Q_{\mathrm{X}}$ is defined as an estimate of the elementary charge, $Q_{\mathrm{X}}=\left.e\right|_{\mathrm{SET}}$, by analogy to the definitions of Josephson and von Klitzing constants, $K_{\mathrm{J}}=2 e /\left.h\right|_{\mathrm{JE}}$ and $R_{\mathrm{K}}=$ $h /\left.e^{2}\right|_{\mathrm{QHE}}[74,75]$. Checking the equality $R_{\mathrm{K}} K_{\mathrm{J}} Q_{\mathrm{X}}=2$ with an uncertainty of one part in $10^{8}$ will be a relevant test of the validity of the three theories. 


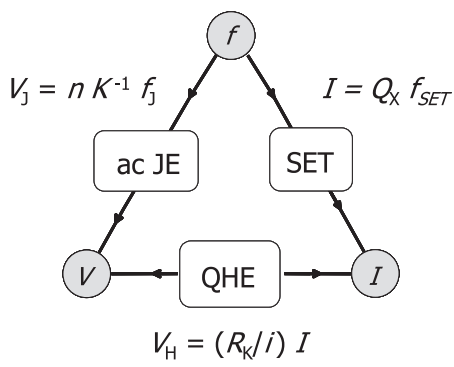

Fig. 9.13 Quantum metrological triangle. Theory predicts that $R_{\mathrm{K}}, K_{\mathrm{J}}$ and $Q_{\mathrm{x}}$ correspond to the fundamental constants $h / e^{2}, 2 e / h$ and $e$.

The principle of the QMT experiment in following Ohm's law rests on the direct comparison of the voltage $V_{J}$ delivered by a Josephson junction array (JJA) biased on the $n$-th Shapiro step to the Hall voltage $V_{H}$ of a QHE sample operating on the $i$-th plateau and fed with a current $I_{1}$ generated by a SET current source ${ }^{10}$. The current $I_{1}$ is amplified by a factor $G_{\mathrm{CCC}}$, the gain of the CCC. From this voltage comparison one finds

$$
V_{\mathrm{J}}=R_{\mathrm{H}}(i) G_{\mathrm{CCC}} I
$$

Taking into account the relationships given by acJE, QHE and SET and using the same notation as in [74], $R_{\mathrm{K}}=h / e^{2}(1+r), K_{\mathrm{J}}=2 e / h(1+k)$ and $Q_{\mathrm{X}}=e(1+q)$, one finds that Eq. (9.28) becomes

$$
s=(n / 2)\left(i / G_{\mathrm{CCC}}\right)\left(f_{\mathrm{J}} / f_{\mathrm{SET}}\right)
$$

where $s=r+q+k$ to first order, and $f_{\mathrm{J}}$ and $f_{\mathrm{SET}}$ are the irradiation and operation frequencies of the JJA and SET devices, respectively. Measurement of the residual term $s$ will give information on the level of consistency of the three quantum phenomena.

An experimental set-up for testing the QMT is sketched in Figure 9.14. The current amplifier is composed of a type I CCC of high winding ratio, $G_{\mathrm{CCC}}=N_{1} / N_{2}$, a dc SQUID with low white noise level and low corner frequency $f_{c}$, and a secondary current source, servo-controlled by the SQUID in such a way that the latter works at null magnetic flux [74]. In order to minimize the contribution from $1 / f$ flicker noise, the polarity of the current to be amplified is periodically reversed. The Hall voltage is simultaneously compared to the voltage of a programmable Josephson junction array voltage standard (JAVS), well suited here because of the low voltage level and the requirement of periodic reversal of polarity [77]. The null detector will be balanced by adjusting the operating frequency of the SET source, $f_{\mathrm{SET}}$. This

10) Another approach consists of balancing the current delivered by the SET device against the current applied to a cryogenic resistor of high resistance (100 M $\Omega$ ) by a Josephson voltage. The current is detected by a CCC operating as a SQUID ammeter, the primary winding being the input coil coupled to the SQUID via a flux transformer [76]. Then the same CCC is used for calibrating directly the $100 \mathrm{M} \Omega$ resistance with the QHRS [47]. 


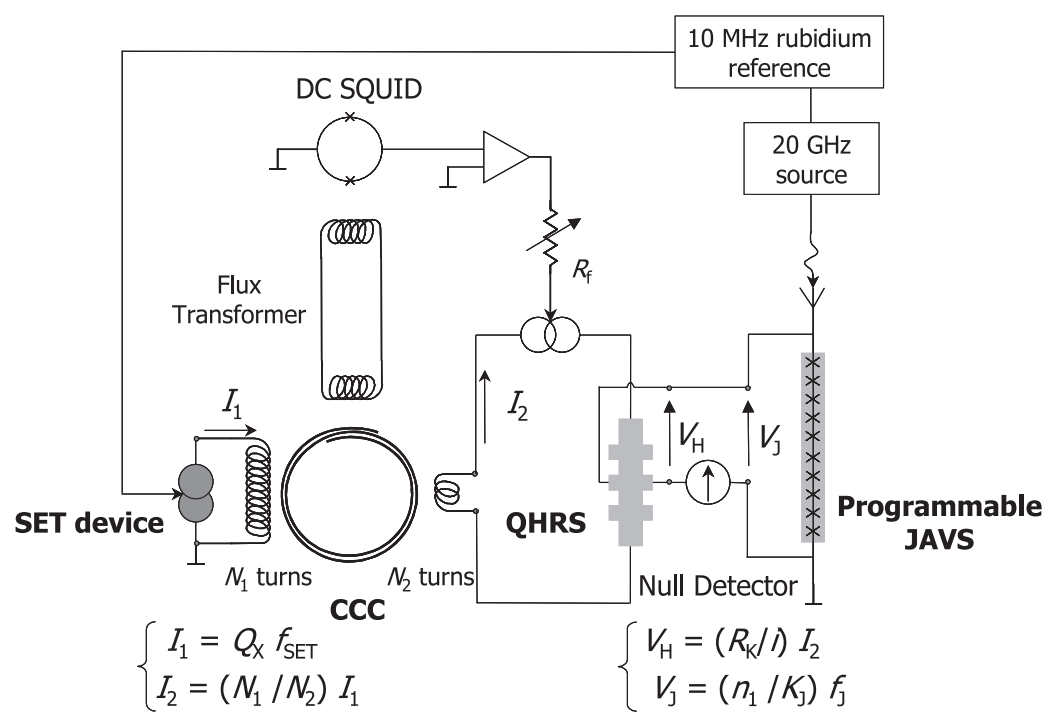

Fig. 9.14 Basic circuit for closing the quantum metrological triangle.

frequency and the irradiation frequency of the JJA are both referred to a $10 \mathrm{MHz}$ rubidium clock.

The great challenge of this experiment is to reduce the "random" uncertainties ${ }^{11}$ to the lowest possible level, one part in $10^{6}$ or maybe less, taking into account the current of a few picoamps delivered by the electron pumps which are at present assumed to be the most accurate current sources. Indeed, Keller et al. have demonstrated that single electrons can be pumped very accurately, i.e., with an uncertainty of one part in $10^{8}$, at frequencies of a few megahertz [78].

The QMT has been succesfully closed within an uncertainty of one part in $10^{6}$ at the National Institute of Standards and Technology (NIST) by constructing an electron counting capacitance standard (ECCS) [72]. This method consists of charging with an electron pump a cryogenic capacitor of known capacitance calibrated in terms of $R_{\mathrm{K}}$ from a QHRs. The voltage drop across the capacitance is controlled with a Josephson array voltage standard. The uncertainty of one part in $10^{6}$ is mainly due to the calibration of a conventional commercial bridge used for measuring the capacitance ratio between the cryogenic capacitor and a room temperature capacitor. In the near future, the uncertainty on the closure of the QMT by this method could be reduced down to one part in $10^{7}$. We note that developments of ECCSs are in progress at other NMIs (METAS, NPL, PTB) ${ }^{12)}$.

11) The largest uncertainties in the instruments used (uncertainties related here to errors coming from systematic effects) are estimated to be of the order of one part in $10^{8}$, and depend weakly on current level. They arise from the finite open loop feedback gain and from leakage currents.

12) METAS (Metrologie und Akreditierung Schweiz) and PTB (Physicalisch Technische Bundesanstalt) are the NMIs of Switzerland and Germany, respectively. 


\subsubsection{Design and Performance of the CCC as an Ultra-Low-Current Amplifier}

The first way to amplify currents as low as a few picoamps with good accuracy is a CCC with very high winding ratio and with a large inner radius to maximize its inductance. In his pioneering work, Hartland developed a 109 999:1 ratio CCC with an overall diameter of $160 \mathrm{~mm}$ leading to a theoretical current resolution $\delta I=80 \mathrm{aA} / \mathrm{Hz}^{1 / 2}[79]$. However, this CCC has never operated properly. One suggested problem is the effect of instability of the helium bath temperature. Another problem might be due to self-resonance. Actually, a compromise on the size of the $\mathrm{CCC}$ and on the number of turns of its windings has to be found. The larger these two parameters are, the better the current resolution, but the stronger the influence of external magnetic fields and the lower the self-resonance frequency. The problem relating to the magnetic field is not insurmountable. It can be solved by enhancing the efficiency of the shielding system. For example, the CCC can be enclosed inside two concentric superconducting shields, the inner shield of niobium and the outer shield of lead, the overall system being located in a closed ferromagnetic cylinder. The theoretical attenuation of such a shielding system is $210 \mathrm{~dB}$ [34].

However, the self-resonance problem is difficult to overcome, especially when it occurs at low frequency, in the bandwidth of the SQUID. For the CCCs of high winding ratio reported below, self-resonances have been observed at frequencies down to a few kilohertz due to the large inductance of the primary winding (of the order of 1 to $10 \mathrm{H}$ ) combined with stray or shunt capacitance (about $100 \mathrm{pF}$ to $1 \mathrm{nF}$ ) [35, 45-47]. This has to be compared with the self- resonance frequencies higher than $30 \mathrm{kHz}$ observed for CCCs of small number of turns (2000 turns at most), such as those used for resistance measurements. A solution to damp the resonating LC oscillations consists of providing the CCC with a resistive primary winding, for example of phosphor-bronze wire [47].

Subsequently, several operating CCCs have been developed. Two of them are coupled to a commercial dc SQUID. One is a 40960:1 ratio CCC with an overall diameter of $64 \mathrm{~mm}$; a current resolution of $\delta I=800 \mathrm{aA} / \mathrm{Hz}^{1 / 2}$ has been measured as the white noise floor. The $1 / f$ noise occurs at the corner frequency $f_{\mathrm{c}}=0.3 \mathrm{~Hz}$ [80].

The other is a $20000: 2$ ratio CCC with a smaller diameter, $46 \mathrm{~mm}$. It achieves a current resolution of $\delta I=4 \mathrm{fA} / \mathrm{Hz}^{1 / 2}$ in the white noise domain and a corner frequency $f_{\mathrm{c}} \approx 0.5 \mathrm{~Hz}$ [34]. The ability to measure currents from $1 \mathrm{pA}$ to a few 
femtoamps with a Type A uncertainty ${ }^{13)}$ of 50 aA (one standard deviation estimate) over a one hour period has been demonstrated with this CCC [35].

A 45 000:1 ratio CCC coupled to a commercial dc SQUID is still in development at the Laboratoire National de Métrologie et d'Essais (LNE) [82]. With an overall diameter of $114 \mathrm{~mm}$, its current resolution is expected to reach $\delta I \approx 100 \mathrm{aA} / \mathrm{Hz}^{1 / 2}$ at $1 \mathrm{~Hz}[83]$.

This CCC is placed in a temperature-stabilized enclosure at $1.5 \mathrm{~K}$. This enclosure is attached to the bottom part of the dilution refrigerator allowing direct access to the SET device. A more compact CCC will be easier to use at very low temperature, for example by placing it inside a dilution unit very close to the SET device. Based on this idea and the proposal of Seppä et al. for a thin-film CCC [84], a CCC with windings based on microlithographic superconducting paths of $2 \mu \mathrm{m}$ width and spaced by the same width is being investigated at LNE.

A fourth CCC is directly and optimally coupled to a home-made low noise dc SQUID [45,46]. This CCC is provided with a $30000: 1$ winding ratio and its overall diameter is $100 \mathrm{~mm}$. The current resolution corresponds to a white noise floor at about $\delta I=2.1 \mathrm{fA} / \mathrm{Hz}^{1 / 2}$ over a frequency range down to a surprisingly low corner frequency, $f_{\mathrm{c}}=0.1 \mathrm{~Hz}$.

A second approach to developing a large-gain CCC while designing it with a small size, as suggested by Sesé et al., is to use a strip-wound ferromagnetic core which increases the inductance of the overlapping tube and maximizes its coupling to the pickup coil [85]. Unlike several earlier reports that the sensitivity of a ferromagnetic core-based CCC is limited by the SQUID's sensitivity, the authors demonstrated that the sensitivity can be preserved or enhanced by introducing a pickup coil with fractional turn loops. However, the dominant $1 / f$ noise due to the ferromagnetic core remains too large (the best material currently used is an amorphous CoNiFe alloy) so that there are no realistic chances for improvement.

\subsubsection{Measurements of SET Current Sources}

A few laboratories have been able to carry out measurements of current delivered by SET devices directly by means of a CCC. For instance, the current delivered by a SETSAW device has been measured at NPL by means of a 40960:1 ratio CCC [80]. However, despite the low uncertainties achieved (3 fA for a $1 \mathrm{nA}$ current), measurements are currently being made using a commercial electrometer, cali-

13) Following well established guidelines, for instance the "GUM" [81] which are recommended for properly expressing and estimating the uncertainties of measurements, there are two categories of statistical methods for evaluating the uncertainties, classified as Type A and Type B. Indeed, it is more suitable to distinguish methods of evaluation rather than uncertainties (by separating them into "random" and "systematic" uncertainties depending on whether the errors come from random and systematic effects) which could lead to ambiguity [82]. The uncertainty evaluated by a Type A method is obtained from a probability density deduced from observed distribution of data. The standard deviation is given by the root square of the usual variance calculated on repeated sets of observations. In contrast to the Type A uncertainties, the uncertainties evaluated by a Type B method are obtained from an assumed probability density based on some level of confidence that an event occurs. 
brated to $15 \mathrm{fA}$ for the $2 \mathrm{nA}$ range, accurate enough for testing the SETSAW device.

LNE has reported the first measurements on SET transistors [86] and SET pumps [87] with a 10000:1 ratio CCC. For these measurements, the SQUID is flux-locked by feeding the current to its modulation coil. By means of a simplified bridge, this internal feedback mode allows one to characterize the SET devices, to measure the noise properties of the complete system with sufficient signal-tonoise ratio and, as reported below, to verify the feasibility of operating SET devices over long periods. In this mode, the gain of the CCC is no longer equal to $N_{1} / N_{2}$ but much smaller and is given by the relation

$$
G_{\mathrm{CCC}}=\left(M_{\mathrm{i}} / M_{\mathrm{f}}\right)(k / 2)\left(L_{\mathrm{CCC}} / L_{\mathrm{i}}\right)^{1 / 2} N_{1}
$$

In practice $G_{\mathrm{CCC}}$ amounts to 5800 with a primary winding of $N_{1}=10000$ turns compared with a 10000:1 ratio in the usual external feedback mode.

In the first experiments with SET transistors, well-defined Coulomb oscillations (peaks of $200 \mathrm{fA}$ ) have been recorded by voltage biasing the transistor with only $100 \mathrm{nV}[83,86]$. For SET pump experiments, flat and extended current steps have been observed at frequencies even as high as $30 \mathrm{MHz}(I=4.8 \mathrm{pA})$ [87]. Preliminary current measurements have been carried out in the electron pumping mode over periods of 30 minutes to 12 hours. An unexpected excess noise was revealed, reaching a level of $130 \mathrm{fA} / \mathrm{Hz}^{1 / 2}$ compared with the CCC noise of $4 \mathrm{fA} / \mathrm{Hz}^{1 / 2}$ (see Figure 9.15). This has led to, for instance, Type A uncertainties of around two parts in $10^{4}$ for the longest measurement time, 12 hours [83].

We note that SET devices operate at very low temperature $(<100 \mathrm{mK})$ where the thermal fluctuation energy of electrons is negligible compared with the Coulomb energy. In order to prevent any assisted photon tunneling processes [88], it is strongly recommended that one filters out any microwave radiation coming from the rest of the system (kept at higher temperature) [83], particularly radiation generated by the Josephson junctions of the SQUID [87]. This requires specific cables

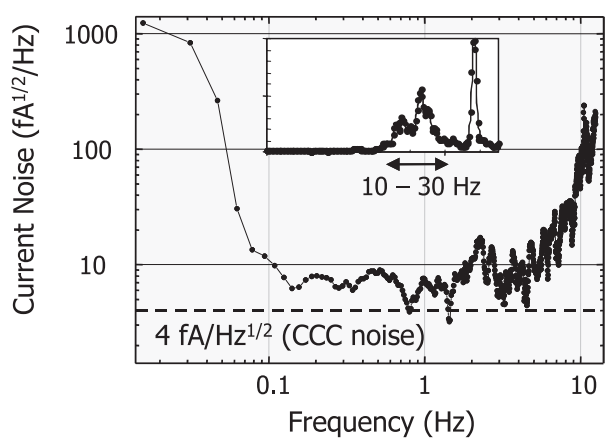

Fig. 9.15 Current noise spectrum of a CCC with an electron pump connected. The insert shows peaks observed around $10 \mathrm{~Hz}$ due to environment mechanical vibrations [87]. 
(some are commercially available) and microwave filters described in detail elsewhere $[86,89]$.

The measurement time for low currents has to be particularly long in order to reduce the random uncertainties. Special attention has to be paid to the nature of the noise. If the condition of uncorrelated noise is fulfilled, Type A uncertainties may be estimated by calculating the experimental standard deviation of the mean of all data [81]. Statistical methods may be helpful, for example Allan variance analysis, which is widely used in the time and frequency domains and also in the field of electrical metrology [90, 91].

The fact that a SET pump can operate over a time as long as 12 hours as shown by these first measurements is very promising $[83,85]$. This confirms the stability time for the background charges (whose change affects the pump accuracy) reported by Keller et al. [78]. They found a stability time between one hour and a few tens of hours on a 7-junction pump, which, however, was not connected to an external circuit (including coil, resistor, filter etc.) contrary to the case in [83, 85].

This stability permits one to expect a reduction of uncertainties down to a few tens of attoamps with a similar 10000:1 ratio CCC. In particular, the standard deviation of the mean given by

$$
\sigma=\delta I\left[\left(f_{\mathrm{S}}-f_{\mathrm{R}}\right) / N\right]^{1 / 2}
$$

where $2 N$ is the total number of current reversals over this measurement time, may reach $20 \mathrm{aA}$, for $\delta I=4 \mathrm{fA} / \mathrm{Hz}^{1 / 2}$, a repetition frequency $f_{\mathrm{R}}$ of a single measurement (two current reversals) equal to the corner frequency $f_{\mathrm{c}}=0.5 \mathrm{~Hz}$ and, in the optimal case, a frequency twice that of the sampling, $f_{\mathrm{S}}=1 \mathrm{~Hz}$. Improved CCCs, combined with new SET devices able to supply quantized current at least ten times higher than existing SET pumps, should make the closure of the metrological triangle feasible with an uncertainty level of few parts in $10^{8}$.

\subsection{5}

\section{Type II CCC for Resistance Measurements at Very High Currents}

The flexibility of the CCC concept is illustrated by the high measurement accuracy (achieving a relative uncertainty $\sigma<10^{-7}$ ) of high currents (up to $100 \mathrm{~A}$ ) in the calibration of resistors down to values of $100 \mu \Omega$ [23]. Here, the type II CCC configuration has the advantage over type I that the current-carrying windings may be positioned further away from the superconducting shield. This is required since the surface magnetic field of the high-current-carrying wires could be large enough to exceed the lower critical field $H_{\mathrm{c} 1}$ of the superconducting shield. In turn this could lead to flux penetration or trapped flux movement, both of which would lead to errors in the CCC current ratio.

CCCs are normally optimized for sensitivity. However, in this high-current application sensitivity to current changes in the windings is not the dominant issue. Instead, there is a risk that background noise in the high-current source will be too large to allow stable SQUID operation. The authors, therefore, deliberately 
reduced the SQUID sensitivity by introducing a short loop of superconducting wire in parallel with the SQUID input.

The dimensions of the CCC are shown in Figure 9.5, where it is indicated how the current-carrying windings may be relatively simply changed to allow changes in ratio or of conductor material. Indeed it is easy to wind and rewind over a toroidal shield. For currents up to $1 \mathrm{~A}$ superconducting NbTi wire $(0.125 \mathrm{~mm}$ in diameter) is used whereas for currents up to $100 \mathrm{~A}$ six $\mathrm{Cu}$ wires are used in parallel, each with a diameter of $0.6 \mathrm{~mm}$. The hatched region within the superconducting shield represents the region occupied by the $\mathrm{Nb}$ wire pickup coil. The ends of this coil are taken out through superconducting shields concentric to the SQUID.

The prototype system has a sensitivity of $S_{\mathrm{CCC}}=13 \times 10^{-6}$ ampereturn $/ \Phi_{0}$, with a current detection level of $\delta I / I=2 \times 10^{-8} / \mathrm{Hz}^{1 / 2}$. The limiting accuracy for the calibration of low value resistors is currently determined by the temperature coefficient of the resistors themselves, which sets a practical limit of $\sigma \approx 4 \times 10^{-7}$.

\subsection{6}

\section{CCC for Non-Invasive Sensing of Charged Particle Beams}

Another metrological application of the CCC aims at real-time non-invasive measurement of charged particle beams in the range $10 \mathrm{pA}-10 \mathrm{~mA}$. Apart from applications in fields such as high energy accelerators, ion implantation and other charged particle beam instruments [92] this use is directed in the longer term at a precision measurement of the Faraday constant $F$ which relates to the SI definition of the amount of substance and the Avogadro constant $N$ via the relationship

$$
N=F / e
$$

Investigations at NPL [27] into HTS current comparator designs, including a readout SQUID also made from HTS material, are aimed at producing a system capable of good accuracy non-invasive measurement of an ion beam current in the range $1 \mu \mathrm{A}$ to $10 \mathrm{~mA}$, such as could be integrated with the isotopically pure $\mathrm{Au}$ ion beam of the type being used for a Faraday constant measurement at PTB [93]. A tube coated with HTS material, inside and out, forms the CCC. A charged particle beam flows along the axis of the tube and the induced screening currents flow on the inside and outside surfaces; the outer current generates a magnetic field which may be sensed by an external SQUID. Again, as in the other CCC applications, the external field is extremely insensitive to the distribution of the charged particle beam over the cross-section of the tube. The mechanical inflexibility and problems with joining currently available HTS materials means that the design of HTS CCC and readout SQUID are severely constrained: only single-layer systems are feasible with existing technology. A schematic of the prototype device is shown in Figure 9.16.

A further important advantage, not apparently demonstrated in conventional superconductor CCCs, is gained when the tube (which is itself made from an insulating material) is coated both inside and outside by a continuous supercon- 


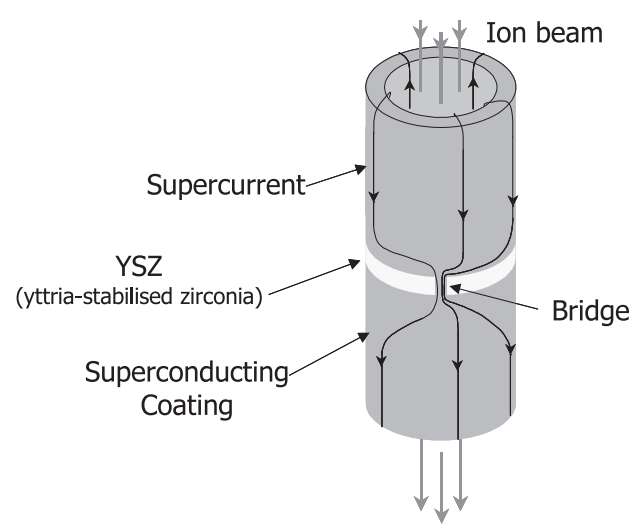

Gradiometric Bridge

Fig. 9.16 HTS CCC based on YBCO - yttria-stabilized zirconia (YSZ) coated tube coupled to a SQUID gradiometer. The size of the CCC developed at $\mathrm{NPL}$ is length $=120 \mathrm{~mm}$, inner diameter $=20 \mathrm{~mm}$, outer diameter $=29 \mathrm{~mm}$.

ducting film. The outer superconducting layer can then be patterned into a narrow bridge structure crossing its median plane, through which a current will flow equal to the entire beam current (as indicated diagrammatically in Figure 9.16). This region of high current density can provide greatly improved coupling to a SQUID detector located near the bridge, so that the sensitivity is no longer limited by the need to locate the detector at a distance from the beam which is usually dictated by the tube diameter. The only limits on the dimensions of this bridge are that, first, the total current flowing through it must not exceed the critical current density $j_{c}$ of the superconducting bridge. A second limit arises when the inductance of the bridge exceeds the inductance of the two-layer superconducting tube in which case current will tend to flow up the inside surfaces of the comparator tube, rather than through the bridge, thereby reducing the sensitivity. Preliminary experiments suggests that $\delta I$ may be as small as $10 \mathrm{pA} / \mathrm{Hz}^{1 / 2}$ for simple single-layer HTS structures.

\section{4}

\section{Other Current Metrological Applications of SQUIDs}

\subsection{1}

\section{Thermometry Using SQUIDs}

\subsubsection{Introduction}

Temperature is an unusual physical parameter in that it is not an extensive quantity. Thermodynamic temperature is defined in terms of two fixed points: the absolute zero of temperature $(0 \mathrm{~K})$ and the triple point of water (defined to be $273.16 \mathrm{~K})$. There exist a number of primary thermometers which are able to sense 
thermodynamic temperatures, but can usually operate over only a limited range of temperature. Thus the best known thermodynamic thermometer is the ideal gas scale. There exists also an International Temperature Scale (ITS-90); it extends down to $0.65 \mathrm{~K}$ and is in close agreement with thermodynamic temperatures throughout its range. A number of different thermometers and fixed points are used to realize the scale. SQUIDs may also be used to indicate either thermodynamic temperatures (through the use of the Josephson effects or magnetic flux fluctuations, in various manifestations of primary noise thermometry) or very small temperature changes.

\subsubsection{Magnetic Susceptibility Measurement for a Secondary Thermometer}

There are at least two quite distinct forms of sensitive temperature sensors based on SQUIDs. The first measures the change of susceptibility $\chi(T)$ with the temperature of a paramagnetic system coupled to a SQUID, where the Curie-Weiss law dependence of the susceptibility

$$
\chi(T)=C_{\mathrm{s}} /\left(T-T_{\mathrm{c}}\right)
$$

allows very small changes to be detected. Here $C_{\mathrm{s}}$ is a constant dependent on the paramagnetic system used and $T_{\mathrm{c}}$ is the ordering temperature of the dilute spins. This system can be used over a relatively wide temperature range. The method may be extended into the millikelvin temperature range by using very dilute paramagnetic salts (cerium magnesium nitrate $(\mathrm{CMN})$ or lanthanum-diluted CMN, for which $T_{\mathrm{c}}<2 \mathrm{mK}$ ) by means of a SQUID-based mutual inductance bridge.

It has long been recognized that for a system with heat capacity $C$ there is a Gaussian probability distribution for the mean square temperature fluctuations given by

$$
\left\langle(\Delta T)^{2}\right\rangle=k_{\mathrm{B}} T^{2} / C_{\mathrm{s}}
$$

When this is converted to a power spectral density $S_{T}(f)$ of temperature fluctuation in unit bandwidth it is transformed to

$$
S_{T}(f)=4 k_{\mathrm{B}} T^{2} / G
$$

where now $G$ is the thermal conductance to a heat sink controlled at $<T>$. This sets a lower limit on the temperature precision which can be achieved with a single measurement within unit bandwidth. Note that the uncertainty scales as $T^{2}$ and that the thermal link between thermometer and heat bath should be as strong as possible in order to minimize temperature fluctuations. Readout sensitivity of such a high-resolution thermometer based on a SQUID is a few $\mathrm{pK} / \mathrm{Hz}^{1 / 2}$, easily capable of measuring $\Delta T$ for real systems, which is more typically $100 \mathrm{pK} / \mathrm{Hz}^{1 / 2}$, so the reality of this practical limit to temperature measurement has been demonstrated [94]. 


\subsubsection{The SQUID as a Sensor for a Resistance Thermometer}

A second method of measuring temperature senses the change in resistance of a thin film superconductor, which is biased (and maintained by negative feedback) at the mid-point of its superconducting transition. The SQUID senses the small change in feedback current which occurs when the temperature of the thin film changes. Since the width of a metallic-superconductor transition can be much less than $1 \mathrm{mK}$, and a change corresponding to a very small fraction of this width is readily detectable (because of the SQUID's ability to detect sub femtovolt voltage changes), this is an extremely sensitive technique, though of much more limited temperature range. It is clearly related to the bolometric sensor application described in Chapter 8.

\subsubsection{Noise Measurement for a "Primary" Thermometer}

Nyquist's theorem states that the power spectral density of noise voltage $S_{V}$ across a resistor $R$ at temperature $T$ (in the classical regime) is given by the simple expression

$$
S_{V}=4 k_{\mathrm{B}} T R
$$

In principle, this provides a method to measure temperature based only on a determination of noise, a simple resistance measurement and knowledge of Boltzmann's constant $k_{\mathrm{B}}$. Thermometers based on this expression have been developed mainly for high temperature use, but it turns out that the necessary characterization of the system bandwidth and gain are too difficult to allow the required millikelvin precision at ambient temperatures. However, in the cryogenic regime the extreme sensitivity of SQUIDs may be used to good effect. In this case, the low input impedance of the SQUID means that a more sensitive thermometer may be developed by using the expression for the current noise spectral density $S_{I}$ associated with a resistance $R$ at temperature $T$

$$
S_{I}=4 k_{\mathrm{B}} T / R
$$

Early work by Webb et al. showed that measurements made between $5 \mathrm{mK}$ and $4.2 \mathrm{~K}$ agreed within $3 \%$ with a magnetic temperature scale derived from the susceptibility of a dilute paramagnetic material [95]. This thermometer does not provide a primary thermometric method since the bandwidth of the SQUID and the flatness of the current gain across the entire bandwidth are required to be known. Rather, it provides a sensitive cryogenic thermometer which may provide rapid measurements down to the lowest temperatures. More recent work by Lusher et al. has further improved on the performance [96].

Kamper and Zimmerman suggested that the subtlety of the Josephson effects could provide a true SQUID-based primary noise thermometer [97]. A Josephson junction is shunted by the low resistance $R$ (in the range $1 \mu \Omega$ to $1 \mathrm{~m} \Omega$ ), forming a resistive SQUID configuration. The essential feature of their proposal was that 
the thermally generated voltage fluctuations across the resistor could be used to frequency modulate an oscillating Josephson junction. The observed Lorentzian lineshape $\Delta f$ of the Josephson oscillation could be accurately measured to provide a measure of the temperature, assuming that $R$ can be accurately measured. A simple and fast way to measure the linewidth of the oscillation is to feed the output from the amplifier to a frequency counter capable of measuring the count variance. In this way, the need to characterize accurately the gain and bandwidth of an amplifier is avoided. In fact the linewidth involves the flux quantum thus:

$$
\Delta f=4 \pi k_{\mathrm{B}} T R / \Phi_{0}^{2}
$$

The circuit for such a measurement system is shown schematically in Figure 9.17(a).

a)

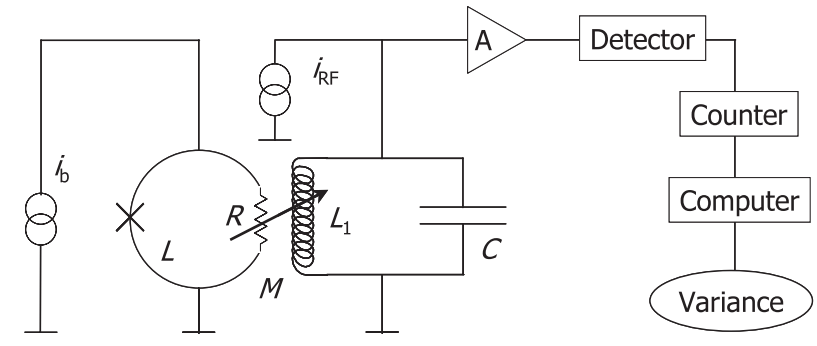

b)

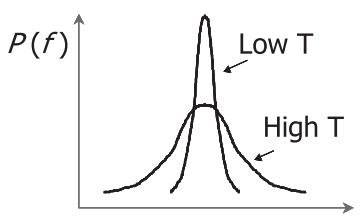

Frequency $f$

Fig. 9.17 Noise measurement system for a "primary" thermometer.

(a) Schematic of Josephson line width thermometer with rf SQUID readout. (b) Schematic of probability distribution of Josephson oscillation frequency at low and high temperatures.

This behavior has been accurately verified and compared with other primary thermometers at NIST and forms the basis of one of two primary thermometers available for use below $1 \mathrm{~K}$ [98]. A second type of noise thermometer based on flux quantization and superconductivity has also been demonstrated by Lee et al. [99]. This is the so-called quantum-roulette noise thermometer in which the probability distribution of the quantized fluxon states of a superconducting ring is experimentally established by repeatedly interrupting the superconducting circuit of the ring and measuring the thermal fluctuation current. A SQUID, magnetically coupled to the superconducting ring, reads out the flux state of the ring following each opening and closing of the switch, allowing a histogram of states to be built 
up. The width of this probability distribution in units of flux quanta allows the temperature to be deduced [99]. Such a single-entity thermometer requires that the principle of ergodicity is satisfied, that is, the long-time average over an arbitrary function of the phase space coordinates is equal to the average of the same function taken over all possible and equally probable states that the system can go through. This principle is becoming increasingly significant in a variety of fields of temperature measurement, especially where the size of the systems is being reduced towards the nanoscale. When the number of individual entities (such as atoms or molecules) in the system falls below a length-dependent number, the definition of temperature itself becomes problematic. As well as providing a potentially valuable primary noise thermometer using high-temperature superconductors, the quantum-roulette noise thermometer provides a test-bed for investigations of the applicability of this principle of ergodicity.

\subsection{2}

\section{Radio-Frequency Attenuation with SQUIDs}

An rf SQUID pumped (biased) at microwave frequency $f$ presents an impedance which is a function, periodic in $\Phi_{0}$, of the instantaneous magnetic flux $\Phi_{\mathrm{a}}$ applied to the SQUID ring. This property can be used for calibrating attenuators at radio frequencies.

In the ideal case and for a non hysteretic SQUID, i.e., one characterized by a small critical current $I_{0}<\Phi_{0} / 2 \pi L$, operating in the non-adiabatic regime $(f>$ $R / 2 \pi L)$, the output voltage of the SQUID is a perfect sinusoidal function of $\Phi_{\mathrm{a}}$ :

$$
V_{\text {out }}\left(\Phi_{\mathrm{a}}\right)=V_{0} \sin \left(2 \pi \Phi_{\mathrm{a}} / \Phi_{0}\right)
$$

Similarly, the power reflected by the SQUID will also vary as a sinusoid:

$$
P\left(\Phi_{\mathrm{a}}\right)=P_{\text {offset }}+P_{0} \sin \left(2 \pi \Phi_{\mathrm{a}} / \Phi_{0}\right)
$$

Consider now an applied flux $\Phi_{\mathrm{a}}(t)=\Phi_{\mathrm{a}} \cos \left(2 \pi f_{\mathrm{a}} t\right)$ varying in time at a frequency $f_{\mathrm{a}}$ much less than the pumping frequency (typically $f_{\mathrm{a}} \approx f / 10$ ), but much greater than the cut-off frequency of the detection circuit bandwidth. It is easy to show that the time-averaged reflected power is given by

$$
P=P_{\text {offset }}+P_{0} J_{0}\left(2 \pi \Phi_{\mathrm{a}} / \Phi_{0}\right)
$$

where $J_{0}$ is the zeroth-order Bessel function.

As shown in Figure 9.18, the applied flux is created through a mutual inductance by the current $I$ which flows across the attenuator A to be calibrated. The main part of the reflected power will follow the zeroth-order Bessel function of this current $J_{0}\left(2 \pi I / I_{0}\right)$. The detection and counting of the zero crossings of $J_{0}\left(2 \pi I / I_{0}\right)$ allow one to calculate rf current ratios and thus to calibrate the attenuator accurately. The zero crossings of the Bessel function are detected by supplying 


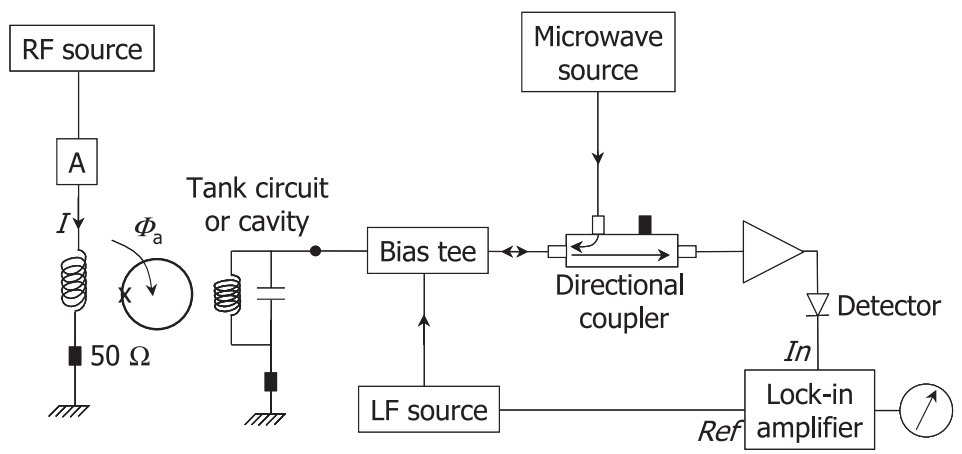

Fig. 9.18 Basic circuit for $\mathrm{rf}$ attenuation measurement with microwave pumped SQUID.

the SQUID with a low-frequency $(1 \mathrm{kHz})$ signal and by recording the zeros at the output of a phase-locked amplifier.

Several decades ago, NMIs used such methods to calibrate rf attenuators [100103]. The microwave-pumped SQUID typically consisted of an adjustable point contact mounted in a superconducting waveguide close to its short-circuited end at a distance of a quarter of a wavelength ${ }^{14}$. The best results were an uncertainty of $0.001 \mathrm{~dB}$ with a dynamic range of more than $100 \mathrm{~dB}$ at frequencies from 0 to $1 \mathrm{GHz}$. Based on these past experiences with this first generation of microwave pumped SQUIDs, two possible sources were known to create deviations of the instantaneous output voltage of the SQUID from a perfect sinusoid, and thus linearity errors in the measured attenuation. The first possibility was the poor quality of the junction itself, which resulted in an inhomogeneous critical current and, therefore, caused departures from the Josephson relation linking the supercurrent to the phase through a perfect sinusoid.

The second cause was the influence of the measurement signal power on the tunnel resistance of the quasiparticles, i.e., the resistance $R$ of the Josephson junction described by the RCSJ model. This effect becomes significant at high frequencies [102]. Moreover the routine of attenuation measurement was compromised by unreliability of the junction.

The drawbacks above could be solved today with a better understanding of SQUID devices and with improved technology for junction and SQUID fabrication. The use of microwave-pumped SQUIDs ${ }^{15}$ for improving attenuation measurement with an uncertainty less than $0.001 \mathrm{~dB}$ could be reconsidered as an alternative to the method presently used, which involves the waveguide beyond cut-off (WBCO) attenuator as primary standard.

14) Adjustable point contacts (in niobium or tantalum) were preferably used rather than microbridges, for which some results reported significant error in attenuation, partly due to the bad thermal contact of the junction with the helium bath.
15) This will probably be limited to LTS SQUIDs since high-temperature (cuprate) SQUIDs with grain-boundary junctions have been shown to exhibit a nonsinusoidal Josephson relationship [104]. 


\section{5}

\section{Future Trends and Conclusion}

This chapter has demonstrated that the best developed and most widely used applications of SQUIDs to metrology occur in the fields of electrical measurements (over a range of frequency and scales) and thermometry (at cryogenic temperatures). This may not always remain the case. It has been observed that metrology (encompassing both precision measurement and standards) provides the earliest applications of many areas of new physics. Examples of this include not only the Josephson effects and the QHE but also many non-SQUID-related innovations in physics such as laser cooling of atoms and the Bose-Einstein condensation. Below we discuss what the authors regard as potentially important future metrological applications of SQUIDs.

As SQUIDs allow the uncertainty principle dominated regime to be investigated, the relationship between quantum measurement fundamentals and quantum metrology comes into focus. At the same time the requirement to make precise measurements at ever smaller length scales initiated a paradigm shift in the nature of electrical (and other) metrology. Thus, as has been made clear above, when SET devices are involved the electrical parameter to be measured is no longer the continuous classical quantity - the electrical current - but rather the quantized electrical charge. Similarly in weakly superconducting electronic circuits it is the passage of single flux quanta which represents the basic electrical measurement not the classical continous variable which is voltage. Metrology must address the needs of the quantum and nanoscale regimes and SQUIDs are well placed to enable the transition, as we shall see below.

With the increasing importance of the spin component of electron transport for future devices (giant and colossal magnetoresistance effects (GMR, CMR), spin valves, magnetic tunnel junctions, etc.) the capability of the SQUID to detect the smallest level of spin polarization will require optimization. Here, the requirements for SQUIDs are the opposite to those for magnetic field sensing: the SQUID loop directly senses the spin and its area must be minimized, subject to optimal coupling to spins.

The most common applications of SQUIDs have involved measurement of magnetic flux density changes with extreme sensitivity. In such cases, it is important to maximize the sensing area of the input coil coupled to the SQUID. In an increasingly important range of newer applications, relating mainly to spintronics and quantum computing, the SQUID is used to detect magnetization or magnetization changes in a micrometer- or sub-micrometer-size sample. In these cases a quite different set of conditions applies [105-107]. There are two major issues which have to be considered in order to develop further such devices and extend their applicability to the study of samples of nanoscale dimension. Firstly, achieving the required sensitivity for the detection of a low number of spins and secondly, facilitating the deposition of such a small sample within the loop of the SQUID. This will enable many of the standards applications outlined in previous sections to be transferred to the nanoscale in future. 
Ultimately, the SQUID may enable single electronic spin flips to be detected, allowing electronic spin resonance (ESR) and other spin manipulations to be done on a single spin. (Recently single spin detection has been achieved using a mechanical magnetic resonance detection device [108] but this technique is rather specialized and of limited applicability). As well as setting the lower limiting size of a single magnetic domain storage element, single spins are also regarded as one of the most promising possible realizations of the qubits on which quantum computation will depend, if it becomes feasible. In such a single-spin detection situation the input coil and flux transformer are dispensed with and a "bare" SQUID is used. Reducing the loop area of the SQUID, which reduces its inductance, can increase the energy resolution of the device to near quantum limit operation. Furthermore, a device incorporating a SQUID of small loop area is less sensitive to external magnetic fields, making it an ideal probe of samples situated within the SQUID loop. In the thermal limit, the energy resolution of a SQUID, of capacitance $C$ and inductance $L$, operating at a temperature $T$ is given by [109]

$$
\varepsilon=16 k_{\mathrm{B}} T(L C)^{1 / 2}
$$

The electronic spin resolution is given by

$$
S_{n}=d \frac{\sqrt{S_{\Phi}}}{2 \pi \mu_{\mathrm{B}} \mu_{0}}
$$

in units of spins (of moment $\mu_{\mathrm{B}}$ ) per $\sqrt{\mathrm{Hz}}$, where $S_{\Phi}$ is the power spectral density of flux noise, related to the energy resolution by $S_{\Phi}=2 \varepsilon L$, and $d$ is the dimension of the SQUID loop, scaled by a geometry-dependent factor between 1 and 10. In measurements made on a set of $\mathrm{Nb} d \mathrm{~d}$ SQUID devices ranging from $d=100 \mu \mathrm{m}$ down to $3 \mu \mathrm{m}$ it has been demonstrated that the flux noise resolution exhibits the expected linear scaling with $d$. The smallest device has a spin resolution of about 40 spins in a $1 \mathrm{~Hz}$ bandwidth limited by noise in the first-stage room temperature preamplifier. Extension of this scaling to SQUIDs with $d \approx 100 \mathrm{~nm}$ should enable single electronic spin flips to be detected. This work has been extended recently by Jamet et al. to barely submicrometer SQUIDs (made using long Dayem bridge $\mathrm{Nb}$ Josepshon junctions) to investigate the magnetic anisotropy of nanoscale particles containing just $10^{3}$ atoms of Co [110].

The overall aim of the QMT experiments is to verify with a very low uncertainty the coherency of the deduced values of the constants involved in these three quantum phenomena or, in other words, to confirm that these condensed matter physics phenomena, in which interparticle interactions are undoubtedly strong, yield the free space values of constants $2 e / h, h / e^{2}$ and $e$. The target uncertainty needs to be around one part in $10^{8}$. If there is no deviation, our confidence in the three phenomena to provide us with $2 e / h, h / e^{2}$ and $e$ will be considerably enhanced. Any significant discrepancy will prompt further experimental and theoretical work. The closure of the QMT, at the required uncertainty of one part in $10^{8}$ or less, should be assisted by improvements in SQUIDs, microlithography techniques and new SET devices which could generate accurate currents as high as 
100 pA. Ideas currently under investigation for the implementation of a higherfrequency-locked current source include improved SETSAW devices [111] and superconducting Cooper pair pumps as a generalization of a single electron pump [112].

Very recently, a Cooper pair "sluice" has been proposed. It consists of two mesoscopic SQUIDs forming between them a superconducting island which is fitted with a gate [113] (Figure 9.19). The gate provides the possibility of coherent transfer of Cooper pair charges, one at a time, under the influence of an applied rf signal, with higher accuracy and at higher frequency than has been demonstrated with electron pumps, turnstiles or SETSAW devices. The closure of the QMT to enhance our confidence of the acJE, QHE and SET providing the ratios of fundamental constants $h / e^{2}, 2 e / h$ and $e$ can be viewed as a bridge between microscopic and macroscopic physics [114]. This bridge is needed to establish a new structure of the SI units, fully based on fundamental constants. It is not clear when we can expect this new formulation of the SI to be implemented. However, it can be expected that SQUIDs will play an important role in these developments.

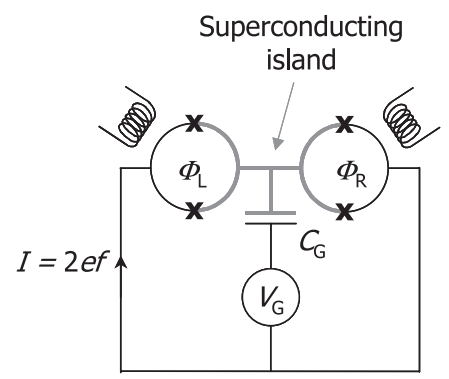

Fig. 9.19 Schematic of a Cooper pair sluice. The flow of Cooper pairs is controlled by varying periodically both the gate voltage $V_{\mathrm{G}}$ applied to the island via a capacitor $\left(C_{\mathrm{G}}\right)$ and the magnetic fluxes $\Phi_{R}$ and $\Phi_{L}$ through the SQUID loops. The superconducting island is shown as a thick gray line. 


\section{References}

1 Clarke, J. (1966) A superconducting galvanometer employing Josephson tunnelling, Phil. Mag. 13, 115-127.

2 Clarke, J. (1968) An experimental comparison of the Josephson voltage frequency relation in different superconductors, Phys. Rev. Lett. 21, 1566-1569.

3 Jain, A. K., Lukens, J. E., and Tsai, J. S (1987) Test for relativistic gravitational effects on charged particles, Phys. Rev. Lett. 58, 1165-1168.

4 Tsai, J. S., Jain, A. K. and Lukens, J. E., (1983) High precision test of the universality of the Josephson voltage-frequency relation, Phys. Rev. Lett. 51, 316-319.

5 CIPM (1988) Représentation du volt au moyen de l'effet Josephson [Representation of the volt by means of Josephson effect], Recommandation 1 (CI - 1988), $77^{\text {ème }}$ session, (1988).

6 Mohr, P. J., and Taylor, B. N., (2005) CODATA (Committee on Data for Science and Technology) recommended values of the fundamental physical constants: 2002, Rev. Mod. Phys. 77, 1-107.

7 Pöpel, R. (1992) The Josephson effect and voltage standards, Metrologia 29, $n^{\circ} 2,153-174$.

8 Hamilton, C. A. (2000) Josephson voltage standards, Rev. Sci. Instrum. 71, 3611-3623.

9 Eichenberger, A., Jeckelmann, B., and Richard, P., (2003) Tracing Planck's constant to the kilogram by electromechanical method, Metrologia 40, 356-365.

10 Shiota, F., Miki, Y., Namba, A., Nezu, Y., Sakamoto, Y., Morokuma, T., and Hara, $\mathrm{K}$, (1995) Absolute determination of the magnetic flux quantum using superconducting magnetic levitation, IEEE Trans. I\&M 44, 583-586.

11 Shiota, F., Miki, Y., Fuji, Y., Morokuma, T., and Nezu, Y. (2000) Evaluation of equilibrium trajectory of superconducting magnetic levitation system for the future $\mathrm{kg}$ unit of mass, IEEE Trans. I\&M 49, 1117-1121.

12 Frantsuz, E. F., Gorchakov, Y. D., and Khavinson, V. M. (1992) Measurements of the magnetic flux quantum, Planck constant, and elementary charge using superconducting magnetic levitation, IEEE Trans. IQM 41, 482-485.

13 Riski, K., Heikkinen, P., Kajastie, H., Manninen, J., Rossi, H., Nummila, K., Frantsuz, E., and Khavinson, V. (2001) Design of a superconducting magnetic levitation system, in Proc. IMEKO TC3 2001, pp.239-246.

14 Sachslehner, F. (1998) Two-channel DCSQUID picovoltmeter for the measurement of low-field Hall coefficient and electrical resistance from 4.2 to $100 \mathrm{~K}$, Cryogenics 38, 293-298.

15 Delahaye, F., and Bournaud, D. (1991) Low noise measurements of the quantized Hall resistance using an improved cryogenic current comparator bridge, IEEE Trans. I\&M 40, 237-240.

16 Miklich, A. H., Koelle, D., Ludwig, F., Nemeth, D. T., Dantsker E., and Clarke, J. (1995) Picovoltmeter based on a high transition temperature SQUID, Appl. Phys. Lett. 66, 230-232.

17 Eriksson, T. Blomgren, J., and Winkler, D, (2002) An HTS SQUID picovoltmeter used as preamplifier for Rogowski coil sensors, Physica C 368, 130-133.

18 Dziuba, R. F., Field, B. F., and Finnegan, T. F. (1974) Cryogenic voltage comparator system for $2 e / h$ measurement, IEEE Trans. I\&M 23, 264-267.

19 Harvey, I. K. (1976) Cryogenic ac Josephson effect emf standard using a superconducting current comparator, Metrologia 12, 47-54.

20 Harvey, I. K. (1972) Precise low temperature DC ratio transformer, Rev. Sci. Instrum. 43, 1626-1629.

21 Sullivan, D. B., and Dziuba, R. F. (1974) Low temperature direct current comparator, Rev. Sci. Instrum. 45, 517-519.

22 Grohmann, K., Hahlbohm, H. D., Lübbig, H., and Ramin, H. (1973) Construction principles and properties of ironless DC and AC current comparators with superconducting shields, PTB Mitt. 83, 313-318.

23 Williams, J. M., and Kleinschmidt P. (1999) A cryogenic current comparator 
bridge for resistance measurements at currents of up to $100 \mathrm{~A}$, IEEE Trans.

I\&M 48, 375-378.

24 Elmquist, R. E. (1999) Cryogenic current comparator measurements at $77 \mathrm{~K}$ using thallium-2223 thick-film shields, IEEE Trans. I\&M 48, 383-386.

25 Elmquist, R. E., and Dziuba, R. F. (1995) High-temperature superconductor cryogenic current comparator, IEEE Trans. I\&M 44, 262-264.

26 Arri, E., Boella, G., Pavese, F., Negro, A., Vanolo, M., Daginino, C., and Lamberti, P. (1998) Improved studies on an HTS current cryocomparator with thickfilm YBCO toroidal shield, in Proc. CPEM'98, Washington DC, pp. 221222.

27 Hao, L., Macfarlane, J. C., and Gallop, J. C. (1999) Ion beam measurement with a high-temperature superconductor SQUID and current comparator, IEEE Trans. I\&M 48, 302-305.

28 Early, M. D., Jones, K., Staines, M. P., and Exley, R. R. (1999) Development of a split-toroid high-temperature superconducting cryogenic current comparator, IEEE Trans. I\&M 48, 306-309.

29 Early, M. D., and Jones, K. (1997) Optimum sensitivity of an externally shielded cryogenic current comparator, IEEE Trans. I\&M 46, 459-462.

30 Frantsuz, E. T. (1998) On forward estimate of the sensitivity of a cryogenic current comparator, Metrologia 35, 847-851.

31 Early, M. D., and van Dam, M.A. (1999) Results from a detailed calculation of the sensitivity of a cryogenic current comparator, IEEE Trans. I\&M 48, 379-382.

32 Sesé, J., Lera, F., Camon, G. and Rillo, C. (1999) Calculation of effective inductances of superconducting devices. Application to the cryogenic current comparator, IEEE Trans. Appl. Supercon, 9, 58-62.

33 Rillo, C., Sesé, J., Bartolomé, E., Flokstra, J., Camon, A., and Rietveld, G. (2003) On the sensitivity of cryogenic current comparators : theory and experiments, Metrologia 40, 51-56.

34 Gay, F., Piquemal, F., and Genevès, G. (2000) Ultra low noise current amplifier based on a cryogenic current comparator, Rev. of Sci. Instrum. 71, 4592-4595.

35 Gay, F., (2000) Un comparateur cryogénique de courants pour la réalisation d'un étalon quantique basé sur l'effet tunnel monoélectron [Cryogenic current comparator for the realisation of a quantum standard based on single electron tunneling], PhD Thesis, Conservatoire National des Arts et Métiers, Paris, France (in French).

36 Symm, G. T. (1992) Design of a cryogenic current comparator, in Proc. Boundary Elements XIV, 1, Brebbia, C. A., Dominguez, J., and Paris, F. (Eds), pp. 519-526.

37 Sesé, J., Bartolomé, E., Flokstra, J., Rietveld, G., Camon, A., and Rillo, C. (2003) Simplified calculus for the design of a cryogenic current comparator, IEEE Trans. I\&M 52, 612-616.

38 Sesé, J. (1999) Optimacion del patron cuantico de resistencia [Optimisation of the quantum resistance standard], $P h D$ Thesis, Saragossa University, Spain, in Spanish.

39 Grohmann, K., Hahlbohm, H.D., and Hechtfischer, D. (1979) The cryocurrent comparator as a calculable dc ratio standard, IEEE Trans. I\&M 28, 205-211.

40 Seppä, H. (1990) The ratio error of the overlapped-tube cryogenic current comparator, IEEE Trans. I\&M. 39, 689-697.

41 Delahaye, F., and Reymann, D. (1985) Progress in resistance ratio measurements using a cryogenic current comparator at LCIE, IEEE Trans. I\&M 34, 316-319.

42 Grohmann, K., and Hechtfischer, D. (1984) Self calibrating cryo current comparators for ac applications, IEEE Trans. I\&M 33, 91-96.

43 Seppä, H., and Satrapinski, A. (1997) AC resistance bridge based on the cryogenic current comparator, IEEE Trans. I\&M 46, 463-466.

44 Delahaye, F. (1991) An ac-bridge for low frequency measurements of the quantized Hall resistance, IEEE Trans. I\&M 40, 883-888.

45 Bartolomé, E. (2002) Cryogenic current comparators with optimum SQUID readout for current and resistance quan- 
tum metrology, PhD Thesis, Twente University, The Netherlands.

46 Rietveld, G., Bartolomé, H., Sesé, J., de la Court, P., Flokstra, J., Rillo, C., and Camon, A. (2003) 1:30 000 cryogenic current comparator with optimum SQUID readout, IEEE Trans. I\&M 52, 621-625.

47 Elmquist, R. E., Hourdakis, E., Jarret, D. G., and Zimmerman,, N. M. (2005) Direct resistance comparisons from the QHR to $100 \mathrm{M} \Omega$ using a cryogenic current comparator, IEEE Trans. I\&M 54, 525-528.

48 Delahaye, F., and Bournaud, D. (1993) Accurate AC measurements of standard resistors between $1 \mathrm{~Hz}$ and $20 \mathrm{~Hz}$, IEEE Trans. I\&M 42, 287-291.

49 Piquemal, F. (1999) L'effet Hall quantique en métrologie [Quantum Hall effect in metrology], Bulletin du BNM 116, 5-57 (in French).

50 Jeckelmann, B., and Jeanneret, B. (2000) The quantum Hall effect as an electrical resistance standard, Rep. Prog. Phys. 64, 1603-1655.

51 Delahaye, F., and Jeckelmann, B. (2003) Revised technical guidelines for reliable dc measurements of the quantized Hall resistance, Metrologia 40, 217-223.

52 Hartland, A., Jones, K., Williams, J. M., Gallagher, B. L., and Galloway, T. (1991) Direct comparison of the quantized Hall resistance in gallium arsenide ans silicon, Phys. Rev. Lett. 66, 969-973.

53 Jeckelmann, B., Inglis, A. D., and Jeanneret, B. (1995) Material, device, and step independence of the quantized Hall resistance, IEEE Trans. I\&M 44, 269-272.

54 Data can be found on BIPM website: http://bipm.fr/.

55 Delahaye, F., Witt, T., Piquemal, F., and Genevès, G. (1995) Comparison of quantum Hall effect resistance standards of the BNM-LCIE and the BIPM, IEEE Trans I\&M 44, 258-261.

56 Delahaye, F., Witt, T., Jeckelmann, B., and Jeanneret, B. (1996) Comparison of quantum Hall effect resistance standards of the OFMET and the BIPM, Metrologia 32, 385-388.

57 Delahaye, F., Witt, T., Pesel, E., Schumacher, B., and Warnecke, P. (1997)
Comparison of quantum Hall effect resistance standards of the PTB and the BIPM, Metrologia 34, 211-214.

58 Delahaye, F., Witt, T., Elmquist, R., and Dziuba, R. F. (2000) Comparison of quantum Hall effect resistance standards of the NIST and the BIPM, Metrologia 37, 173-176.

59 Satrapinski, A., Seppä, H., Schumacher, B., Warnecke, P., Delahaye, F., Poirier, W., and Piquemal, F. (2001) Comparison of four QHR systems within one month using a temperature and pressure stabilized $100 \Omega$ resistor, IEEE Trans. I\&M 50, 238-241.

60 Nakanishi, M., Kinoshita, J., Endo, T., Zhang, Z., Shao, H., He, Q., and Liang, B. (2002) Comparison of resistance standards between the National Institute of Metrology (China) and the Electrotechnical Laboratory (Japan), Metrologia 39, 207-212.

61 CIPM (1988) Représentation de l'ohm au moyen de l'effet Hall quantique [Representation of the ohm by means of quantum Hall effect], Recommandation 2 (CI - 1988), 77ème session.

62 Pesel, E., Schumacher, B., and Warnecke, P. (1995) Resistance scaling up to $1 \mathrm{M} \Omega$ with a cryogenic current comparator, IEEE Trans. I\&M 44, 273-275.

63 Fletcher, N., Janssen, J. T., and Williams, J. (2001) A cryogenic current comparator based resistance bridge for the range $10 \mathrm{k} \Omega$ to $1 \mathrm{G} \Omega$, in Proc. British Electro-Magnetic Conference BEMC'01, Harrogate, UK.

64 Poirier, W., Bounouh, A., Hayashi, K., Fhima, H., Piquemal, F., Genevès, G., and André, J. P. (2002) $R_{\mathrm{K}} / 100$ and $R_{\mathrm{K}} /$ 200 quantum Hall array resistance standards, J. Appl. Phys. 92, 2844-2854.

65 Poirier, W., Bounouh, A., Piquemal, F., and André, J. P. (2004) A new generation of QHARS : Discussion about the technical criteria for quantization, Metrologia 41, 285-294.

66 Hartland, A. (1992) The quantum Hall effect and resistance standards, Metrologia 29, 175-190.

67 Geerligs, L. J., Anderegg, V. F., Holweg, P. A., Mooij, J. E., Pothier, H., Estève, D., and Devoret, M. H. (1990) Fre- 
quency-locked turnstile device for single electrons, Phys. Rev. Lett. 64, 2691-2694.

68 Pothier, H., Lafarge, P., Estève, D., Urbina, C., and Devoret, M. H. (1992) Single electron pump based on charging effects, Europhys. Lett. 17, 249-254.

69 Grabert H., and Devoret M. H (eds.) (1991) Single charge Tunneling Coulomb Blockade Phenomena in Nanostructures, Nato ASI series, New York Plenum Press., B294.

70 Shilton, J. M., Talyanskii, V. I., Pepper, M., Ritchie, D.A., Frost, J. E. F., Ford, C. J. B., Smith, C. G., and Jones, A.C. (1996) High-frequency single-electron transport in a quasi-one-dimensional GaAs channel induced by surface acoustic waves, Journal of Physics: Condensed Matter 8, L531-L539.

71 Likharev, K., and Zorin, A. (1985) Theory of the Bloch-wave Oscillations in small Josephson junctions, J. Low Temp. Phys. 59, 347-382.

72 Keller, M. W., Eichenberger, A. L., Martinis, J. M., and Zimmerman, N. M. (1999) A capacitance standard based in counting electrons, Science 285, 17061709.

73 Keller, M. W. (2001) Standards of current and capacitance based on singleelectron tunneling devices, in Recent Advances in Metrology and Fundamental Constants, FERMI School CXLVI, Varenna, Italy, pp. 291-316.

74 Piquemal, F., and Genevès, G. (2000) Argument for a direct realization of the quantum metrological triangle, Metrologia 37, 207-211.

75 Piquemal, F., Bounouh A., Devoille L., Feltin N., Thevenot O., and Trapon G. (2004) Fundamental electrical standards and the quantum metrological triangle, C. R. Physique 5, 857-879.

76 Elmquist, R., Zimmerman, N. M., and Huber, W. H. (2003) Using a high-value resistor in triangle comparisons of electrical standards, IEEE Trans. I\&M 52, 590-593.

77 Hamilton, C. A., Burroughs, C. J., and Kautz, R. L. (1995) Josephson D/A converter with fundamental accuracy, IEEE Trans. I\&M 44, 223-225.

78 Keller, M. W., Martinis, J. M., Zimmerman, N. M, and Steinbach, A. H. (1996)
Accuracy of electron counting using a 7junction electron pump, Appl. Phys. Lett. 69, 1804-1807.

79 Hartland, A. (1993) Development of a cryogenic current comparator for the measurement of small current, in Proc. British Electro-Magnetic Conference BEMC'93, UK, pp. 18/1-18/4.

80 Janssen, T. B. J. M., and Hartland, A. (2000) Accuracy of quantized single electron current in a one dimensional channel, Phys. B. 284-288, 1790-1791.

81 ISO (1993) Guide to the Expression of Uncertainty in Measurement, International Standardization Organisation (ISO).

82 Following some changes of French metrological organization (2001-2005), the same group reported on its works under successive labels BNM-LCIE (Bureau National de Métrologie - Laboratoire Central des Industries Electriques) then BNM-LNE (Laboratoire National d'Essais) and since 2005 LNE (Laboratoire National de Métrologie et d'Essais).

83 Feltin, N., Devoille, L., and Piquemal, F. (2004) Contribution of LNE within the COUNT project, In COUNT final report, van den Brom, H., Coord., contract no. G6RD-CT 1999-00046 (unpublished).

84 Seppä, H., Satrapinski, A., Kiviranta, M., and Virkki, V. (1999) Thin-film cryogenic current comparator, IEEE Trans. I\&M 48, 365-369.

85 Sesé, J., Camon, A., Rillo, C., and Rietveld, G. (1999) Ultimate current resolution of a cryogenic current comparator, IEEE Trans. I\&M 48, 1306-1313.

86 De Wilde, Y., Gay, F., Piquemal, F., and Genevès, G. (2001) Measurements of single electron transistor devices combined with a CCC: progress report, IEEE Trans. I\&M 50, 231-234.

87 Feltin, N., Devoille, L., Piquemal, F., Lotkhov, S., and Zorin, A. (2003) Progress in measurements of single electron pump by means of a CCC, IEEE Trans. I\&M 52, 599-603.

88 Covington, M., Keller, M. W., Kautz, R. L., and Martinis, J. M. (2000) Noiseinduced leakage and counting errors in the electron pump, Phys. Rev. Lett. 84, 5192-5195. 
89 Vion, D., Orfila, P. F., Joyez, P., Estève, D., and Devoret, M. H. (1995) Miniature electrical filters for single electron devices, J. Appl. Phys. 77, 2519-2524.

90 Allan, D. W. (1987) Should the classical variance be used as a basic measure in standards metrology, IEEE Trans. I\&M 36, 646-654.

91 Witt, T. J. (2000) Testing for correlations in measurements, in Advanced Mathematical and Computational techniques in Metrology IV, Ciarlini, P., Paves, F. and Richter, D., (Eds.), Singapore: World Scientific, pp. 273-288.

92 See for instance: Peters, A., Vodel, W., Koch, H., Neubert, R., Reeg, H., and Schroeder, C. H. (1998) A Cryogenic Current Comparator for the absolute measurement of $\mathrm{nA}$ beams, AIP Conference Proc. 451, 163-180.

93 Ratschko, D., Zhou, B. G., Knolle, D. and Glaeser, M. (1997) Investigations on ion beams from a high current ion source, IEEE Trans. I\&M 46, 588-591.

94 Day, P., Hahn, I., Talso, C. P., Chui, Harter, A. W., Rowe, D., and Lipa, J. A. (1997) The fluctuation-imposed limit for temperature measurement, J. Low Temp. Phys. 107, 359-370.

95 Webb, R. A., Giffard, R. P., and Wheatley, J. C. (1973) Noise thermometry at ultra low temperatures, J. Low Temp. Phys. 13, 383-429.

96 Lusher, C. P., Junyun, Li, Maidanov, V. A., Digby, M. E., Dyball, H., Casey, A., Nyéki, J., Dmitriev, V. V., Cowan, B. P., and Saunders, J. (2001) Current sensing noise thermometry using a low $T_{\mathrm{C}} \mathrm{DC}$ SQUID preamplifier, Meas. Sci. Technol. 12, 1-15.

97 Kamper, R. A., and Zimmerman, J. E. (1971) Noise thermometry with the Josephson effect, J. Appl. Phys. 42, 132136.

98 Hudson, R. P., Marshak, H., Soulen, R. J., and Utton, D. B. (1975) Recent advances in thermometry below 300mK, J. Low Temp Phys. 20, 1-102.

99 Lee, R. A. M., Macfarlane, J., Romans, E., Ling Hao, Peden, D., and Gallop, J. (2001) Quantum Roulette Noise Thermometer: progress and prospects, IEEE Trans. Appl. Supercond, 11, 859-862.
100 Kamper, R. A., Simmonds, M. B., Adair, R. T., and Hoer, C. A. (1973) A new technique for rf measurements using supercoductors, in Proceedings of IEEE 61, 121-122.

101 Sullivan, D. B., Adair, R., and Frederick, N. V. (1978) RF instrumentation based on superconducting quantum interference, in Proc. IEEE 66, 454-463.

102 Petley, B. W., Morris, K., Yell, R. W., and Clarke, R. N. (1976) Moulded microwave SQUID for RF attenuation calibration, Electron. Lett. 12, 237-238.

103 Seppä, H. (1983) Some new aspects concerning the $\mathrm{X}$ band SQUID for the measurement of RF attenuation, IEEE Trans. I\&M 32, 253-259.

104 Il'ichev, E., Zakosarenko, V., Ijsselsteijn, R., Schultze, V., Meyer, H., Hoenig, H., Hilgenkamp, H., and Mannhart, J. (1998) Nonsinusoidal Current-Phase Relationship of Grain Boundary Josephson Junctions in High-Tc Superconductors, Phys. Rev. Lett. 81, 894-897.

105 Gallop, J. C., and Radcliffe, W. J. (1985) An absolute SQUID magnetometer, IEEE Trans. Magn. 21, 602-605.

106 Ketchen, M. B., Awschalom, D. D., and Gallagher, W. J. et al. (1989) Design, fabrication, and performance of integrated miniature SQUID susceptometers, IEEE Trans. Magn., 25 n², 1212-1215.

107 Narasimhan, L. R., Takigawa M., and Ketchen M. B. (1994) Magnetic resonance of a small platinum particle using an integrated dc SQUID, Appl. Phys. Lett. 65, 1305-1307.

108 Rugar, D., Budakian, R., Mamin, H. J., and Chui, B. W. (2004) Single spin detection by magnetic resonance force microscopy, Nature 430, 329-332.

109 Tesche, C. D., and Clarke, J. (1977) DC SQUID: Noise and Optimization, J. Low Temp. Phys. 29, 301-331.

110 Jamet, M., Wernsdorfer, W., Thirion, C., Mailly, D., Dupuis, V., Mélinon, P., and Pérez, A. (2001) Magnetic Anisotropy of a Single Cobalt Nanocluster, Phys. Rev. Lett. 86, 4676-4679.

111 Fletcher, N. E., Ebbecke, J., Janssen, T. J. B. M., Ahlers, F., Pepper, M., Beere, H. E., and Ritchie, D. A. (2003) Quantized acoustoelectric current transport through a static quantum dot using a 
surface acoustic wave, Phys. Rev. B 68, 245310/1-3.

112 Zorin, A., B., Lotkhov, S. V., Bogoslovsky, S. A., and Niemeyer, J. (2001) Radio-frequency-driven motion of single Cooper pairs across the superconducting single-electron transistor with dissipative environment, cond-mat/0105211.
113 Niskanen, A. O., Pekola, J., P., and Seppä, H. (2003) Fast and accurate single-island charge pump: Implementation of a Cooper pair pump, Phys. Rev. Lett. 91, 177003/1-4.

114 Bordé, C., private communication. 
